NASA Technical Memorandum 109081
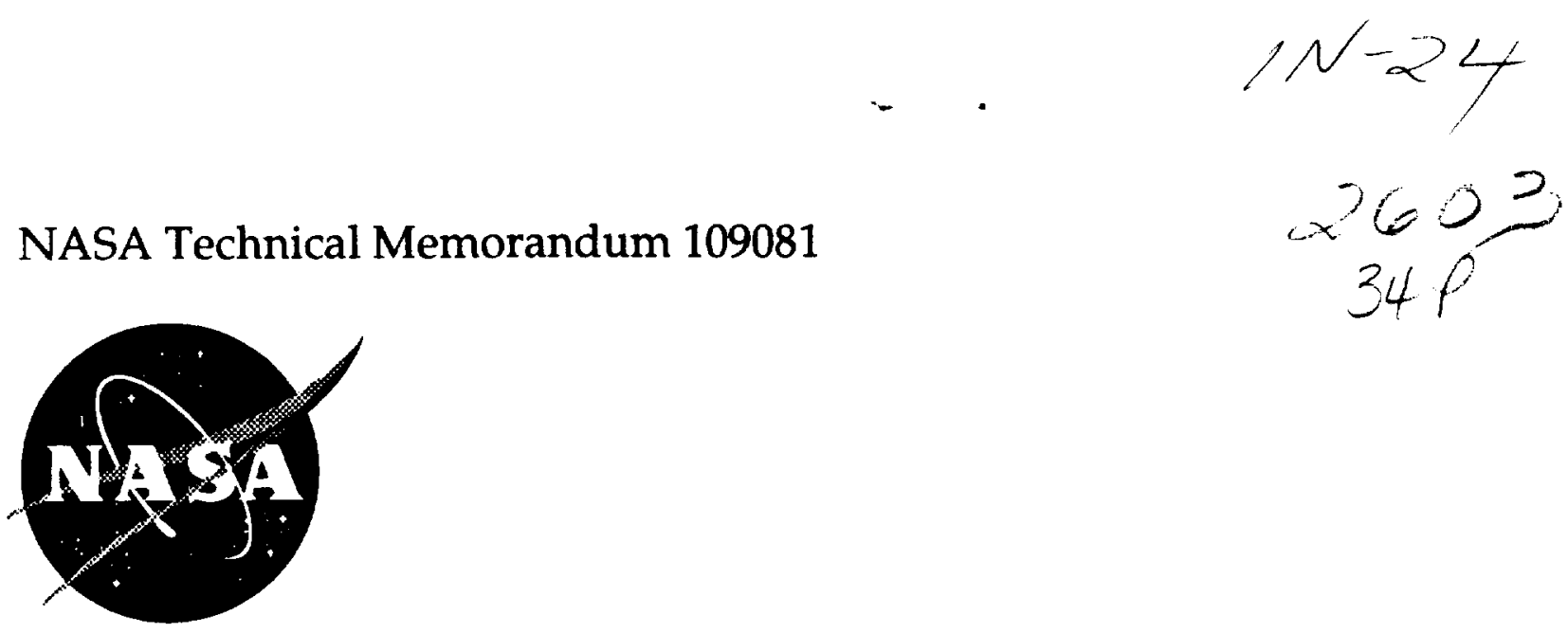

\title{
Effects of Physical Aging on Long-Term Creep of Polymers and Polymer Matrix Composites
}

L. Catherine Brinson

Northwestern University, Evanston, Illinois

Thomas S. Gates

Langley Research Center, Hampton, Virginia

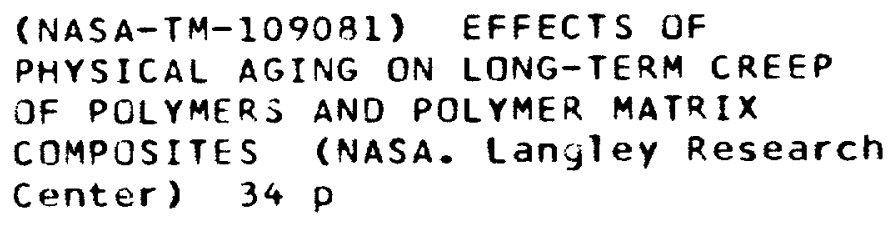

N94-29541

Unclas

$63 / 24 \quad 0002603$

February 1994

National Aeronautics and Space Administration Langley Research Center Hampton, Virginia 23681-0001 
- . 


\section{NOMEnClature}

$a_{t_{e}} \ldots . . . . .$. aging time shift factor, at aging time $t_{e}$

$a_{t_{e}^{0}}(t)$.....aging time shift factor, at aging time $t+t_{e}^{0}$, relative to aging time $t_{e}^{0}$

$a_{t_{\text {erf }}}$.......shift rate at reference aging time, unity by definition

$\bar{a}_{T}$...........time temperature shift factor

$\bar{a}_{T_{1} / T_{2}}^{t_{e_{j}}}$.....time-temperature shift factor between $T_{1}$ and $T_{2}$ at aging time $t_{c j}$

$[A]$.........matrix relating midplane strains to resultant forces in laminate

B............shape parameter

$C_{1}^{0}$..........free volume parameter for WLF equation

$C_{2}^{0}$..........free volume parameter for WLF equation

$\left\{\varepsilon_{x}\right\}$.......vector of strain components $=$ $\left\{\varepsilon_{x x}, \varepsilon_{y y}, \varepsilon_{x y}\right\}$

$\left\{\varepsilon_{x}^{0}\right\}$......strains at the midplane of the laminate

h............thickness of laminate

$\left\{\kappa_{x}\right\}$......vector of curvatures at the midplane of the laminate

$\lambda$.............effective time

H............shift rate

$\mu_{1}$..........shift rate prior to equilibrium aging time

$\mu_{2}$..........shift rate after equilibrium aging time is reached

$\mu_{22}$........shift rate for $S_{22}$

$\mu_{66} \ldots . . . . . . s h i f t$ rate for $S_{66}$

$\left\{M_{x}\right\}$....resultant moments in laminate

$\left\{N_{x}\right\}$.....resultant forces in laminate
$[\bar{P}(t)] \ldots . .$. effective modulus matrix of the laminate in the loading coordinate system

$\theta$............ angle between the fiber direction and the longitudinal loading axis

$[\bar{Q}]$..... inverse of the S-bar matrix for the kth lamina

$S$............creep compliance

$S_{0}$............ initial compliance

$[\bar{s}]$.........effective compliance matrix $S_{22}$........ transverse compliance in $0^{\circ}$ lamina $S_{66}$......... shear compliance in $0^{\circ}$ lamina

क........... constant stress in time $\left\{\sigma_{x}\right\} \ldots . .$. vector of stress components $=$ $\left\{\sigma_{x x}, \sigma_{y y}, \sigma_{x y}\right\}$

$\left\{\bar{\sigma}_{x}\right\} \ldots . .$. average stresses through the thickness in laminate

t............. time

T............. relaxation time

$t_{e}$............ aging time

$t_{e}^{0}$........... initial aging time

$t_{\text {erf }}$........ reference aging time

$t_{e}^{*}$...........equilibrium aging time

[T] ........ transformation matrix based on $\theta$

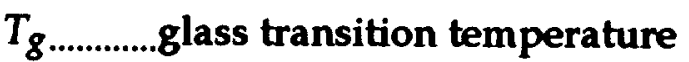

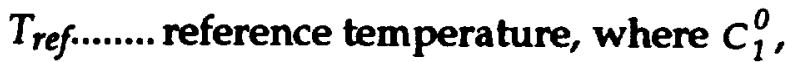
$C_{2}^{0}$ are defined

$z$............. coordinate through the thickness of the laminate 
. 


\section{ghrects of Physical Agivg on long-Twam Creip of Polymers and Polymig Matrx \\ Composimes}

\author{
L. Catherine Brinson \\ Mechanical Engineering Department \\ Northwestern University \\ Evanston, IL 60202-3111
}

\author{
Thomas S. Gates \\ NASA Langley Research Center \\ MS 188E \\ Hampton, VA 23681-0001
}

\section{Abstract}

For many polymeric materials in use below the glass transition temperature, the long term viscoelastic behavior is greatly affected by physical aging. To use polymer matrix composites as critical structural components in existing and novel technological applications, this long term behavior of the material system must be understood. Towards that end, this study applied the concepts governing the mechanics of physical aging in a consistent manner to the study of laminated composite systems. Even in fiber-dominated lay-ups the effects of physical aging are found to be important in the long-term behavior of the composite.

This paper first lays out in a self-consistent manner the basic concepts describing physical aging of polymers. Several aspects of physical aging which have not been previously documented are also explored in this study, namely the effects of aging into equilibrium and a relationship to the time-temperature shift factor. The physical aging theory is then extended to develop the long-term compliance/modulus of a single lamina with varying fiber orientation. The latter is then built into classical lamination theory to predict long-time response of general laminated composites. Comparison to experimental data is excellent. In the investigation of fiber oriented lamina and laminates, it is illustrated that the long term response can be counterintuitive, stressing the need for consistent modeling efforts to make long term predictions of laminates to be used in structural situations. 


\section{Introduction}

The High Speed Civil Transport aircraft (HSCT), envisioned to have a lifetime of over 60,000 flight hours and to travel at speeds in excess of Mach 2, is the source of intensive design and development studies at NASA and major airframe developers. In the area of materials for the HSCT, polymer matrix composites (PMC's) are being strongly considered for use in primary and secondary structures due to their high strength to weight ratio and the options of property tailoring. However, one difficulty in the long term use of polymer based materials is that their strength and stiffness properties may change measurably over time (especially at the elevated temperatures experienced during flight). Analytical prediction of these properties based on irregular thermal and mechanical loading may be extremely difficult.

The current study is focused on one aspect of long-term polymer composite viscoelastic behavior: physical aging. In contrast to the equilibrium state of a polymer above its glass transition temperature, when a polymer is cooled to below its glass transition temperature the material does not achieve instantaneous thermodynamic equilibrium. Instead the free volume, enthalpy and entropy evolve over time toward their hypothetical equilibrium values. During this time the mechanical properties change significantly. This change is termed physical aging. Figure 1 illustrates the change in the volume in a polymer as a function of temperature; when the polymer is cooled below the glass transition temperature, the volume is initially in a nonequilibrium state and evolves slowly towards equilibrium volume with time.

In essence, during physical aging the material becomes stiffer, so that at a given time the compliance is decreased (or the modulus increased) over what one would have expected in a viscoelastic material without aging. In terms of free volume theory (Struik, 1978; Ferry, 1980), one can visualize that as the free volume decreases towards its equilibrium value, the mobility of chain segments is hindered, giving rise to a stiffer response. Aging is a characteristic of the glassy state and is found in all polymer glasses, as thoroughly documented by Struik (1978). The aging 
of polymer composite systems has received little attention until recent years. But several thorough experimental studies (Sullivan, 1990; Gates and Feldman, 1993; Hastie and Morris, 1993; Sullivan, Blais et al., 1993) have illustrated that the matrix dominated composite properties of continuous fiber reinforced PMC's, namely the shear and transverse response, are affected by physical aging in a similar manner to pure polymers.

This work begins with a review of the concepts of physical aging on a pure polymer system and the theory is outlined in a consistent manner. The effective time theory (Struik, 1978; Knauss and Emri, 1981), which can be used to predict long term behavior based on short term data, is also mathematically formalized. The effects of aging to equilibrium are proven and discussed and a special relationship of aging to time-temperature superposition is demonstrated. The physical aging theory developed for polymers is then applied first to a unidirectional composite, then to a general laminate. It will be shown that the effects of aging on the long-term properties of composites can be counter-intuitive, stressing the importance of the development and use of a predictive theory to analyze PMC structures.

\section{Physical Aging of Polymers}

The traditional means to assess and quantify the effects of physical aging on mechanical properties is through a series of sequenced creep (constant load) and recovery tests (Struik, 1978). In these tests, the specimen is initially quenched from above the glass transition $\left(T_{g}\right)$ to a temperature below $T_{g}$. The time the material exists below its glass transition is referred to as the aging time, $t_{e}$. As aging time progresses, a series of short (in comparison to the elapsed aging time) creep tests are run to extract the momentary creep compliance of the material. The momentary creep compliance of the material can be described with a three parameter fit model, first proposed for linear viscoelastic polymers by Kohlrausch (1847),

$$
S(t)=S_{0} e^{(t / \tau)^{\beta}}
$$

where $S_{0}$, is the initial compliance, $\beta$ is a shape parameter, $t$ is time, and $\tau$ is the relaxation time. 
As shown in Figure 2a (and more explicitly in the isochronal plot in Figure $2 \mathrm{~b}$ ), the material is stiffer (or less compliant) at any given test time as aging time progresses. In nearly all cases of aging of polymers and PMC's it is possible to bring the momentary creep curves measured at different aging times into superposition through a horizontal shift. ${ }^{\dagger}$ This result is akin to the wellknown time-temperature superposition principle (Ferry, 1980). If the aging time shift factor, $a_{t_{e}}$, is plotted as a function of aging time on a double-log scale, it is found to map a straight line with a slope of $\mu$ as shown in Figure 3. The shift rate, $\mu$,

$$
\mu=-\frac{d \log a_{t_{e}}}{d \log t_{e}}
$$

usually has values on the order of unity and can be considered to be a material constant. Thus, a series of creep tests (as in Figure 2a) can be used to experimentally determine the value of the shift rate, $\mu$, for any given material.

Since the momentary creep curves superpose through horizontal shifting on the log scale. the only parameter which changes as a function of aging time is the relaxation time. In terms of the mathematical expression of compliance in Equation (1), the momentary material properties then vary with aging time according to

$$
S(t)=S_{0} e^{\left(t / \tau\left(t_{e}\right)\right)^{\beta}}
$$

where

$$
\tau\left(t_{e}\right)=\tau\left(t_{\text {eref }}\right) / a_{t_{e}}
$$

$t_{\text {eref }}$ is the reference aging time, $a_{t_{e r f}}=1$ and the aging time shift factor is given by

t A small vertical shift is also sometimes needed in order to achieve ideal superposition. The full implications of the vertical shift factor in terms of modeling will be reserved for a later investigation. A study of the vertical shifting involved in aging of composites can be found in (Sullivan and Blais et al., 1993).As with all viscoelastic material descriptions and shifting procedures, certain trends have been shown to be valid for a wide range of amorphous polymers (for physical aging, Struik (1978) has compiled exhaustive data) and the equations to describe the behavior are selected based upon this body of empirical data; the constants used in the equations are material properties determined from specific experiments on each polymer individually. 


$$
a_{t_{e}}=\left(\frac{t_{c r e f}}{t_{e}}\right)^{\mu}
$$

Thus to describe the momentary creep compliance at any aging time, the parameters needed are the initial compliance $\left(S_{0}\right)$, the shape parameter $(\beta)$, the shift rate $(\mu)$, and the relaxation time $(\tau)$ at a reference aging time ( $t_{\text {eref }}$ ). Note that if a given aging time is greater than the reference aging time $\left(t_{e}>t_{\text {eref }}\right), a_{t_{e}}<1$; otherwise $a_{t_{e}}>1$. In the case that $t_{e}>t_{e r e f}$, the relaxation time at $t_{e}$ is also greater $\left(\tau\left(t_{e}\right)>\tau\left(t_{e r f f}\right)\right)$ and for any given test time the material is in a "glassier" state such that the compliance is lower and the modulus higher $\left(S\left(t ; t_{e}\right)<S\left(t ; t_{\text {eref }}\right)\right)$.

\section{Effective Time Theory}

If the loading time of a material is not short in comparison to the previous aging time, then aging proceeds even as the test progresses. Thus, if the beginning aging time is $t_{e}^{0}$, at some time later during the test the aging time is $t+t_{e}^{0}$, where $t$ is the test time. When the test time, $t$, approaches and exceeds the value of the initial aging time, one would expect deviation of the long-term creep response from the momentary. Taking the initial aging time $t_{e}^{0}$ to be the reference aging time $\left(t_{e r e f}=t_{e}^{0}\right)$, the shift factor, $a_{t_{e}^{0}}$, at any instant in time can be defined based on $\mu$ :

$$
a_{t_{e}^{0}}(t)=\left(\frac{t_{e}^{0}}{t_{e}^{0}+t}\right)^{\mu}
$$

In this notation, the updated aging time is always greater than the initial aging time and consequently $a_{t_{e}^{0}}<1$.

To account for the cumulative effects of aging, one can consider what happens in any discrete differential time interval, $d t$. During a time increment $d t$ at time $t$ all relaxation times are $1 / a$ slower and the time increment is $1 / a$ less eventful than if aging time had not been increasing. The "effective" time increment can then be defined (Morland and Lee, 1960; Struik, 1978)

$$
d \lambda=a_{t_{e}^{0}}(t) d t
$$

and the total test time can be reduced to the "effective time" (or "reduced" time), $\lambda$ 


$$
\lambda=\int_{0}^{t} a_{t_{e}^{\circ}}(\xi) d \xi
$$

Integration of (8) using (6) leads to two distinct expressions for effective time

$$
\begin{aligned}
& \mu=1 \ldots \lambda=t_{e}^{0} \ln \left(\frac{t}{t_{e}^{\prime \prime}}+1\right) \\
& \mu \neq 1 \ldots \lambda=\frac{t_{e}^{0}}{1-\mu}\left[\left(1+\frac{t}{t_{e}^{0}}\right)^{1-\mu}-1\right]
\end{aligned}
$$

Using the effective time in place of real time in Equation (3)

$$
S(t)=S_{0} e^{\left(\lambda / \tau\left(t_{e}^{0}\right)\right)^{\beta}}
$$

results in prediction of long-term material response (as opposed to the momentary response) based only on viscoelastic material parameters determined from short term tests. Notice that the relaxation time, $\tau$, is calculated based on a reference aging time, $t_{\text {eref }}$, which may differ from $t_{e}^{0}$, in which case the value of $\tau$ is updated on the basis of equations (4) and (5) using $t_{e} \equiv t_{e}^{0}$, not equation (6):

$$
\tau\left(t_{e}^{0}\right)=\tau\left(t_{\text {eref }}\right)\left(\frac{t_{e}^{0}}{t_{\text {eref }}}\right)^{\mu}
$$

At any given test time, $t$, the long term response is mapped from the value of the momentary response at time $\lambda$. Figure 4 illustrates the effective time theory prediction of longterm material response using idealized material properties. Note that the compliance deviates sharply from the exponential momentary curve as the test time approaches and exceeds the initial aging time." If the initial aging time for a long-term test is changed, both $\lambda$ and $\tau$ must be updated based on the shift rate and the new initial aging time, using equations (6) and (9) and

- For the illustrated long-term test, the material response would follow the long-term response curve only, not the momentary curve. Experimental data can be obtained for the upper portion of the momentary curve, however, by performing tests at higher temperatures with the same initial aging dime and using time-temperature superposition to get the momentary master curve. (At.the higher temperature, the upper portion of the momentary curve is actually obtained at shorter times, ie, before $t>t_{e}^{0}$.) 
(11). For $\mu=1$, the long-term curves for different $t_{e}^{0}$ are simply shifted horizontally from one another on the log time scale; for $\mu \neq 1$ this is no longer true.

Figure 5 illustrates the role of the shift rate, $\mu$, in the long-term response of a material. Note that increasing values of $\mu$ lead to a stiffer (less compliant) response at longer times and that a small change in the shift rate can lead to radically different response at long times. Thus the shift rate can be used as a viscoelastic screening parameter for selection of materials. As the shift rate goes to zero, the long-term response approaches the momentary response, so that a shift rate of zero indicates no aging and the long-term curve coincides with the momentary curve for $t_{\text {eref }}$; the creep compliance then increases exponentially with loading time without the retarding effect of aging, a situation undesirable for most PMC structures. As a final note on the significance of the shift rate, in the past shift rates were considered to have an ideal value of unity, but values of less than one were also common. No mention was made of shift rates exceeding one, particularly in the theory, but current data on composites has yielded shift rates exceeding one (Gates and Feldman, 1993). In this respect, it should be noted that the aging and effective time theory is selfconsistent and shift rates exceeding one are both physically and mathematically permissible. As the shift rate increases from a value of unity, the effective time $\lambda$ decreases and if the shift rate were allowed to obtain the physically unrealistic value of infinity, the effective time would be zero and the compliance of the material would equal the initial compliance at all times.

\section{Aging to Equilibrium}

For most polymers, if the material is being loaded at temperatures close to its glass transition, the material can age into effective equilibrium during a relatively short time frame. The time required to achieve equilibrium is known as the equilibrium aging time, $t_{e}^{*}$ (Lee and McKenna, 1990; Santore, Duran et al., 1991). Because the material state upon cooling deviates further from thermodynamic equilibrium as the temperature decreases and because aging proceeds on a log-time basis, the temperature must be very close to $T_{g}$ for equilibrium to be 
reached in a realistic time scale. Such situations are of interest for polymer composite design studies because viscoelastic material properties of an aircraft wing, for example, may vary significantly if equilibrium is reached.

According to experimental evidence on a polymer by (Lee and McKenna, 1990) and preliminary evidence by (Gates and Feldman, 1993) on a composite, when the equilibrium aging time is exceeded the shift rate does not become zero but decreases by an order of magnitude. This might indicate that aging has not completely ceased, such that absolute thermodynamic equilibrium has not been attained. But recalling the sensitivity of the long term modulus to the shift rate, an order of magnitude change in $\mu$ indicates that the rate of aging is indeed greatly reduced. In general, the experimental results showed $\mu_{1} \approx 1$ before $t_{e}^{*}$ and $\mu_{2} \approx 0.1$ after $t_{e}^{*}$ and this is illustrated by the bilinear shift rate in Figure 6 .

It will be shown that the basic concepts of aging and effective time can be mathematically formalized to handle the case of aging into equilibrium. The analysis must simply proceed along lines considering the distinction between an initial aging time that is less than versus one that is greater than the equilibrium aging time. To calculate the momentary compliance at any aging time, for $t_{e}<t_{e}^{*}$ all precepts remains as before and

$$
\tau\left(t_{e}\right)=\tau\left(t_{\text {eref }}\right)\left(\frac{t_{e}}{t_{\text {eref }}}\right)^{\mu_{1}} .
$$

If $t_{e}>t_{e}^{*}$, however, then the calculation of the shift factor (and therefore the relaxation time) must account for the change in shift rate after aging into equilibrium. Expressing $\mu_{2}$ and $\mu_{1}$ as the slopes of the double-log shift factor-aging-time curve (Figure 6),

$$
\mu_{2}=-\frac{\log a_{t_{e}}-\log a_{t_{e}^{*}}^{*}}{\log t_{e}-\log t_{e}^{*}} \quad \mu_{1}=-\frac{\log a_{t_{e}^{*}}-\log a_{t_{\text {eref }}}}{\log t_{e}^{*}-\log t_{\text {eref }}}
$$

and combining and simplifying these equations (noting that $\log a_{t_{\text {enf }}}=0$ by definition), one finds

$$
\log \left(\frac{t_{e}^{*}}{t_{e}}\right)^{\mu_{2}}=\log a_{t_{e}}-\log \left(\frac{t_{e \text { ref }}}{t_{e}}\right)^{\mu_{1}}
$$


From equation (14) the shift factor for $t_{e}>t_{e}^{*}$ is obtained

$$
a_{t_{c}}=\left(\frac{t_{e}^{*}}{t_{e}}\right)^{\mu_{2}} \cdot\left(\frac{t_{e r e f}}{t_{e}^{*}}\right)^{\mu_{1}}
$$

from which one deduces that relaxation time can be expressed as

$$
\tau\left(t_{e}\right)=\tau\left(t_{\text {eref }}\right) \cdot\left(\frac{t_{e}}{t_{e}^{*}}\right)^{\mu_{2}} \cdot\left(\frac{t_{e}^{*}}{t_{\text {eref }}}\right)^{\mu_{1}}
$$

In the calculation of effective time for long-term response predictions, the situation is somewhat reversed. If $t_{e}^{0}<t_{e}^{*}$, then as the test time increases in a long-term test, the aging time increases from its initial value of $t_{e}^{0}$ and at some point will exceed the value of $t_{e}^{*}$. Define this point in time as $\hat{\imath}$

$$
\hat{t}=t_{e}^{*}-t_{e}^{\prime}
$$

The integration for the effective time calculation from equation (8) must be separated then into two parts. For $t<\hat{t}$, the shift factor is as given for equation (6),

$$
a_{t_{e}}(t<\hat{t})=\left(\frac{t_{e}^{0}}{t_{e}^{0}+t}\right)^{\mu_{1}}
$$

but for $t>\hat{t}$ the calculation must proceed along the lines as for equation (15), yielding

$$
a_{t_{e}}(t>\hat{t})=\left(\frac{t_{e}^{*}}{t_{e}^{0}+t}\right)^{\mu_{2}}\left(\frac{t_{e}^{0}}{t_{e}^{*}}\right)^{\mu_{1}}
$$

Combining, the effective time for $t_{e}^{0}<t_{e}^{*}$ is

$$
\lambda=\int_{0}^{\hat{t}}\left(\frac{t_{e}^{0}}{t_{e}^{0}+\xi}\right)^{\mu_{1}} d \xi+\int_{\hat{i}}^{t}\left(\frac{t_{e}^{*}}{t_{e}^{0}+\xi}\right)^{\mu_{2}}\left(\frac{t_{e}^{0}}{t_{e}^{*}}\right)^{\mu_{1}} d \xi
$$

where $a_{t_{e}^{0}}\left(t_{e}^{0}\right)=1$. This can be solved explicitly to yield

$$
\lambda=\frac{t_{e}^{0}}{1-\mu_{1}}\left[\left(1+\frac{\dot{t}_{e}^{*}-t_{e}^{0}}{t_{e}^{0}}\right)^{1-\mu_{1}}-1\right]+\left(\frac{t_{e}^{0}}{t_{e}^{*}}\right)^{\mu_{1}} \cdot \frac{t_{e}^{*}}{1-\mu_{2}}\left[\left(\frac{t_{e}^{0}+t}{t_{e}^{*}}\right)^{1-\mu_{2}}-1\right] .
$$

If $t_{e}^{0}>t_{e}^{*}$, the effective time for long-term response can be calculated much more readily. Again defining $a_{t_{e}^{0}}\left(t_{e}^{0}\right)=1$, Equation (8) applies directly and 


$$
\lambda=\int_{0}^{t}\left(\frac{t_{e}^{0}}{t_{e}^{0}+\xi}\right)^{\mu_{2}} d \xi
$$

Equations (12) and (16) can be used quite accurately to predict the momentary response of the aging data of a polymer or a composite aged to equilibrium (illustrated in Figure $2 \mathrm{a}$, where the curves coincide with experimental data points). Figure 7 shows the results of the long-term predictions using equations (21) and (22) along with the momentary data from (Lee and McKenna, 1990).

In Figure 7 , note that if the material is loaded with an initial aging time less than $t_{e}^{*}$, the response initially follows the momentary curve; then begins to deviate (becoming much stiffer than the momentary curve predicts) as the test time approaches initial aging time; then as the test time and initial aging time combined exceed $t_{e}^{*}$, the material has "aged into equilibrium" and the response follows parallel to the momentary curve once again. Since the material is aging at a much reduced rate after $t_{e}^{*}$, accumulating time no longer has the effect of causing the material to remain much stiffer than the momentary response. In the long term response of a material aged into equilibrium $\left(t_{e}^{0}>t_{e}^{*}\right)$, the compliance increases in an exponential manner. The results are in good agreement with the short term responses at increasing aging times, however there is as yet no experimental data for long term response. From short-term data as shown in Figure $2 a$, after equilibrium the momentary responses do not continue to shift significantly on the horizontal logtime scale. Thus the long term response, which is in essence a compilation of momentary responses at longer and longer aging times, would also not be expected to have the compliance values extend horizontally at a consistent low magnitude across the time scale. These results indicate that due to the dramatic change in material response upon aging into equilibrium, this possibility should be considered during the design stage for structural components of polymers or PMC's, particularly in high-temperature applications. 


\section{Aging Time - Temperature Relationships}

In the course of this study the relationship between aging time and time-temperature superposition was investigated. In general, to obtain the most accurate results for the aging time shift rate it is necessary to run momentary creep tests at various temperatures for each aging time. In this manner, through time-temperature superposition one obtains a larger segment of the momentary master curve and the comparisons of momentary master curves at different aging times to calculate the aging time shift rate is more accurate. A general time/temperature/agingtime shift factor can be expressed (Sullivan, 1990)

$$
\log a=\log a_{t_{e}}+\log \bar{a}_{T}
$$

where $a_{t_{e}}$ is the aging-time shift factor as defined by equation (5) which brings isothermal creep curves at different aging times into superposition and $\bar{a}_{T}$ is the time-temperature shift factor which is generally expressed through the (Williams-Landel-Ferry) WLF equation at temperatures above the $T_{g}$ (Ferry, 1980)

$$
\log a_{T}=\frac{C_{1}^{0}\left(T-T_{\text {ref }}\right)}{C_{2}^{0}+T-T_{\text {ref }}}
$$

where $C_{1}^{0}$ and $C_{2}^{0}$ are material constants based upon free volume. In (24) the bar is deliberately omitted from the $a_{T}$ since above the $T_{g}$ the time-temperature shift factor will be independent of physical aging, as aging does not occur. Unfortunately a theoretically derived equation to represent $\bar{a}_{T}$ below the glass transition temperature is not available, with or without consideration of physical aging effects. A common technique when using time-temperature shifting on experimental data both above and below the $T_{g}$ is to use a WLF fit for data above the $T_{g}$ and to do a linear fit for the data below the $T_{g}$ (Rusch, 1968; Brinson, 1990).

It has been demonstrated that the shift rate which characterizes the aging of a material, although constant for a wide range of temperatures, is in fact a function of temperature (Struik, 1978). In particular near the $T_{g}$ of a material, the shift rate decreases, the value being identically zero above $T_{g}$. Because of this temperature dependence of $\mu$, and thus of $a_{t_{e}}$, then that the time- 
temperature shift factor, $\bar{a}_{T}$, must be a function of aging time below $T_{g}$. It will be shown here that exactly this relationship will allow us to mathematically formalize the dependence of $\bar{a}_{T}$ on aging time, allowing us to predict $\bar{a}_{T}$ at any aging time if we know its value or functionality at a single aging time and we know the temperature dependence of $\mu$.

To see the relationship between $\bar{a}_{T}$ and $\mu(T)$, consider the following four cases outlined schematically in Figure 8: momentary compliance curves at temperature $T_{1}$ for various aging times, momentary compliance curves at temperature $T_{2}$ for various aging times, momentary compliance master curves at $t_{e 1}$ for various temperatures, and momentary compliance master curves at $t_{e 2}$ for various temperatures. Taking $t_{e 1}=t_{e r e f}$, at $T_{1}$ the relationship between compliance at two aging times can be expressed (using equation 5)

$$
S_{t_{e 1}}^{T_{1}}(t)=S_{t_{e 2}}^{T_{1}}\left(t\left(\frac{t_{e 2}}{t_{e 1}}\right)^{\mu\left(T_{1}\right)}\right)
$$

Similarly at $T_{2}$,

$$
S_{t_{e 1}}^{T_{2}}(t)=S_{t_{e 2}}^{T_{2}}\left(t\left(\frac{t_{e 2}}{t_{e 1}}\right)^{\mu\left(T_{2}\right)}\right)
$$

For constant aging time $t_{e 1}$, the relationship between momentary compliance master curves at two temperatures can be given by

$$
S_{t_{e 1}}^{T_{2}}(t)=S_{t_{e 1}}^{T_{1}}\left(t \bar{a}_{T_{1} / T_{2}}^{t_{e 1}}\right)
$$

Similarly at $t_{e 2}$,

$$
S_{t_{e 2}}^{T_{2}}(t)=S_{t_{e 2}}^{T_{1}}\left(t \bar{a}_{T_{1} / T_{2}}^{t_{e 2}}\right)
$$

denoting $\bar{a}_{T_{1} / T_{2}}^{t_{e j}}$ as the time-temperature shift factor between $T_{1}$ and $T_{2}$ at aging time $t_{e j}$. Equating like compliance terms using straight-forward substitution and reduction, one can obtain

$$
\frac{\bar{a}_{T_{1} / T_{2}}^{t_{e 2}}}{\bar{a}_{T_{1} / T_{2}}^{t_{e 1}}}=\left(\frac{t_{e 2}}{t_{e 1}}\right)^{\mu\left(T_{2}\right)-\mu\left(T_{1}\right)} .
$$

Thus, given the shift rate as a function of temperature, $\mu(T)$, and the values or an expression for the time-temperature shift factor at a single aging time, $\bar{a}_{T}$ can be calculated at any 
aging time. Since time-temperature superposition is generally used to collect sufficiently detailed momentary curves at any given aging time, these formal relationships between aging time and time-temperature superposition will be of utility in data reduction.

\section{Aging and Long Term Response of a Composite Lamina (Orthotropic Plate)}

The response of a composite lamina under plane stress conditions is generally described through transformation expressions which relate the properties of the lamina measured along and transverse to the fiber direction with the angle, $\theta$, between the fiber direction and the longitudinal loading axis. (See Figure 9.) In this manner an effective compliance matrix, $[\bar{S}]$, for any off-axis lamina is defined (Jones, 1975; Halpin, 1992) as

$$
[\bar{S}]=[T]^{-1}[S][T]
$$

where

$$
[T]=\left[\begin{array}{ccc}
\cos ^{2} \theta & \sin ^{2} \theta & 2 \sin \theta \cos \theta \\
\sin ^{2} \theta & \cos ^{2} \theta & -2 \sin \theta \cos \theta \\
-\sin \theta \cos \theta & \sin \theta \cos \theta & \cos ^{2} \theta-\sin ^{2} \theta
\end{array}\right] \quad[S]=\left[\begin{array}{ccc}
S_{11} & S_{12} & 0 \\
S_{12} & S_{22} & 0 \\
0 & 0 & S_{66}
\end{array}\right]
$$

The terms in the $S$ matrix are the properties of the lamina with respect to the fiber coordinate system and relate the stress to the strain in the fiber direction. The elastic stress-strain relation for any fiber orientation under in-plane loading is then given by

$$
\left\{\begin{array}{l}
\varepsilon_{x x} \\
\varepsilon_{y y} \\
\varepsilon_{x y}
\end{array}\right\}=[\bar{S}]\left\{\begin{array}{l}
\sigma_{x x} \\
\sigma_{y y} \\
\sigma_{x y}
\end{array}\right\} \text {. }
$$

where $\varepsilon_{i j}$ and $\sigma_{i j}$ represent the strain and stress in the axis directions indicated.

Experimental data on polymer composites (Sullivan, 1990; Hastie and Morris, 1993) has shown that the transverse compliance, $S_{22}$, and the shear compliance, $S_{66}$, are the only terms in the compliance matrix that are dominated by polymer matrix properties. Only these terms are thus time-dependent and subject to physical aging. The transverse and shear compliance depend on the viscoelastic parameters introduced earlier and can be represented by equations (3), (4) and (5), such that in functional form 


$$
\begin{aligned}
& S_{22}(t)=f\left(S_{22}^{0}, \beta_{22}, \tau_{22}\left(t_{\text {eref }}\right), \mu_{22} ; t\right) \\
& S_{66}(t)=f\left(S_{66}^{0}, \beta_{66}, \tau_{66}\left(t_{\text {eref }}\right), \mu_{66} ; t\right)
\end{aligned}
$$

Note that the shear and transverse compliance of a composite are each described independently by the four viscoelastic parameters: initial compliance, shape parameter, a relaxation time at a given reference aging time ${ }^{\ddagger}$, and a shift rate; these parameters are measured in the laboratory for a given composite material in short-time tests. Using equations (3) or (10), (4) and (5) with equations (32) enables the calculation of the momentary or the long-term shear and transverse compliance of a composite lamina with respect to loading in the fiber direction.

In this analysis, we will not consider the full hereditary integral constitutive expression for the viscoelastic behavior (Findley, Lai et al., 1989), but will concentrate on the case of creep compliance from constant load. A general linear viscoelastic constitutive law may be written as

$$
\varepsilon(t)=\int_{-\infty}^{t} S(t-\tau) \frac{d \sigma(\tau)}{d \tau} d \tau
$$

and in a constant load creep test the stress may be represented as

$$
\sigma(t)=\sigma_{0} H(t)
$$

where $H(t)$ is the unit step function. Substitution of (34) into (33) yields

$$
\begin{aligned}
& \varepsilon(t)=\sigma_{0} \int_{-\infty}^{t} S(t-\tau) \delta(\tau) d \tau \\
& \varepsilon(t)=\sigma_{0} S(t)
\end{aligned}
$$

Given a constant load assumption, equation (31) can then be applied directly, substituting the appropriate time-dependent quantities.

₹ Most generally, the reference aging time for the relaxation time can differ for the transverse and the shear compliance. One must then, however, carry a superscript along on the reference aging times for clarity: $t_{\text {erfof }}^{22}$ and $t_{\text {enef. }}^{66}$. 
Thus, the response of a lamina for any angle, $\theta$, of fiber orientation, can be determined through the transformations accounted for in equation (31) together with the expressions for $S_{22}$ and $S_{66}$ and the constant terms of the S-matrix:

$$
\left\{\begin{array}{l}
\varepsilon_{x x}(t) \\
\varepsilon_{y y}(t) \\
\varepsilon_{x y}(t)
\end{array}\right\}=[\bar{S}(t)]\left\{\begin{array}{l}
\sigma_{x x} \\
\sigma_{y y} \\
\sigma_{x y}
\end{array}\right\} ; \quad \bar{S}_{i j}(t)=f\left(\theta, S_{i j}\right)
$$

or in shorthand notation:

$$
\left\{\varepsilon_{x}(t)\right\}=[\bar{S}(t)]\left\{\sigma_{x}\right\} .
$$

For linear viscoelastic behavior, the relaxation times and effective time are updated individually for each of the time-dependent terms and then combined in a consistent manner to yield the overall long-term response. Note again that equation (37) holds only for the case of constant loading; however, the time-dependent compliance matrix itself (but not the stress-strain relation) calculated in this manner holds for any loading.

Note that the $\bar{S}$-matrix will generally be fully populated and all of the terms will be timedependent, in contrast the $S$-matrix. Consequently, due to the complex interaction of all these terms and the independent time parameters ( $\mu$ and $\tau$ ) of $S_{22}$ and $S_{66}$, the long-term compliance of a lamina with a given fiber angle cannot be intuitively predicted.

To illustrate the complexity of long term lamina response, consider the following example using IM7-8320 data (Table 1) (Hastie and Morris, 1993). In Figure 10 the long term load-direction compliance of a composite lamina at various fiber angles is presented as predicted by the theory outlined in this paper. The results coincide precisely with experimental data (not shown) up to $10^{6}$ seconds, after which there are no further experimental data points. Note that at all times the $90^{\circ}$ lamina is the most compliant, the $0^{\circ}$ the least compliant, and the remaining angles falling in order in between, as one might expect. To easily compare the relative change over time of the compliance with respect to initial value for the different angles, Figure 11 shows the same results shifted down uniformly to the $0^{\circ}$ curve. In this figure, one sees that the long time response does 
not consistently follow a "simple" rule that the $0^{\circ}$ compliance changes the least (none) with time, all others increasingly more so to a maximum with the $90^{\circ}$ lamina.

Tabb 1: Transverseand Shear Compliance Data on M7-8320 system (Hastie and Moris, 1993)

\begin{tabular}{|c|l|l|}
\hline VE parameters: & \multicolumn{1}{|c|}{$S_{22}$} & \multicolumn{1}{|c|}{$S_{66}$} \\
\hline$\mu$ & 0.77 & 0.93 \\
$\beta$ & 0.416 & 0.456 \\
$\tau$ & $1.19 \times 10^{6} \mathrm{sec}$ & $4.31 \times 10^{5} \mathrm{sec}$ \\
$S_{0}$ & $750 \times 10^{-9} \mathrm{~Pa}^{-1}$ & $1364 \times 10^{-9} \mathrm{~Pa}^{-1}$ \\
$t_{\text {eref }}$ & $3.24 \times 10^{4} \mathrm{sec}$ & $3.24 \times 10^{4} \mathrm{sec}$ \\
\hline \hline Elastic parameters: & $S_{11}=40.23 \times 10^{-9} \mathrm{y} / \mathrm{psi}$ & $v_{12}=0.348$ \\
\hline
\end{tabular}

If one notes that in Figures 10 and 11, the shift rates for $S_{22}$ and $S_{66}$ are quite different ( $\mu_{66}=0.93, \mu_{22}=0.77$ ), an immediate conclusion is that since the shift rates are different for those two terms, the aging rates and thus effective time aiso differ. Such a discrepancy will be manifested in different rates of change of the long-term compliance for different fiber angles, where the relative influence of $S_{22}$ or $S_{66}$ is determined through the transformation equation (30). The complex coupling of the extension and shear behavior is clearly illustrated by artificially imposing a constraint of equal shift rates. With such a constraint, it might be expected that a "simple" rule for change of compliance over time would be met. However, as is illustrated in Figures 12 and 13, if $\mu_{66}=\mu_{22}=0.77$ the result is that even if we assume that the rates of aging are the same, the long term response at various fiber angles definitely do not follow any simple rule. In fact, in this case for very long times, the compliance of a $45^{\circ}$ lamina exceeds that of a $90^{\circ}$ lamina. And if the curves are again shifted uniformly to the $0^{\circ}$ curve it is clear that the compliance of the $30^{\circ}$ lamina in fact changes the most over time and that the $90^{\circ}$ changes the least of all except for the $0^{\circ}$ lamina.

In this second case (Figures 15 and 16) where we have artificially forced equal shift rates (and equal effective time) for the transverse and shear compliance, one clearly sees the influence of another time factor. The relaxation times for the $S_{22}$ and $S_{66}$ compliance terms also directly 
affect the long-time response of the lamina. Since these relaxation times in general differ between $S_{22}$ and $S_{66}$, and the relative influence of $S_{22}$ versus $S_{66}$ in the load direction compliance is different for every fiber angle via equations (30) and (37), even if the shift rates are the same it cannot be expected that the time-dependent response follow a simple pattern. In this particular case, the $S_{66}$ relaxation time is an order of magnitude smaller than for $S_{22}$ at the same reference aging time, indicating that the $S_{66}$ exponential will have a larger magnitude for lower values of time than $S_{22}$. Thus, for hypothetically equal shift rates, a lamina with fiber angle of $30^{\circ}$, which is strongly influenced by $S_{66}$, is predicted to change more over time than the $90^{\circ}$ lamina which is influenced only by $S_{22}$.

Conversely, when the shift rates are allowed to assume their real values, since $\mu_{66}$ has a higher magnitude than $\mu_{22}$ the effect of physical aging will be such that the rate of increase of the $S_{66}$ compliance with time is substantially more dampened than the $S_{22}$ compliance. The opposing effects of the shorter relaxation time and the higher shift rate then combine to yield a response that is closer over the long term for $S_{22}$ and $S_{66}$ than expected. An illustration of this is given in Figures 14 and 15, where the individual compliance terms are plotted for the two shift rate cases.

\section{Aging of a Laminate}

The next logical step in the analysis is to examine the response of composite laminates to physical aging. This can be accomplished by incorporating the process of physical aging of a lamina (as developed in the previous section) into standard lamination theory. In this study, the theory for thin laminates was used and the results presented are for symmetric laminates with inplane loading only, although the theory applies readily to non-symmetric laminates and more general loading. From classical plate theory, the stress-strain relation for the kth lamina in a composite is given by (Jones, 1975; Halpin, 1992)

$$
\left\{\sigma_{x}\right\}_{k}=[\bar{Q}]_{k}\left\{\left\{\varepsilon_{x}^{0}\right\}+z\left\{\kappa_{x}\right\}\right\},
$$


where $\left\{\sigma_{x}\right\}_{k}$ represents the stresses in the kth lamina, $[\bar{Q}]_{k}$ is the inverse of the S-bar matrix (as defined by equations (30) and (37) for the kth lamina, $\left\{\varepsilon_{x}^{0}\right\}$ are the strains at the midplane of the laminate, $z$ is the coordinate through the thickness and $\left\{\kappa_{x}\right\}$ is the vector of curvatures at the midplane of the laminate. Upon introduction of the resultant forces $(N)$ and moments $(M)$ in a laminate containing $n$ lamina with an overall thickness, $b$,

$$
\begin{gathered}
\left\{N_{x}\right\}=\int_{-h / 2}^{h / 2}\left\{\sigma_{x}\right\} d z=\sum_{k=1}^{n} \int_{z_{k-1}}^{z_{k}}\left\{\sigma_{x}\right\}_{k} d z \\
\left\{M_{x}\right\}=\int_{-h / 2}^{h / 2}\left\{\sigma_{x}\right\} z d z=\sum_{k=1}^{n} \int_{z_{k-1}}^{z_{k}}\left\{\sigma_{x}\right\}_{k} z d z
\end{gathered}
$$

and substitution of equation (38), one obtains a system of equations which can be solved to determine the state of stress and strain at any point in the laminate:

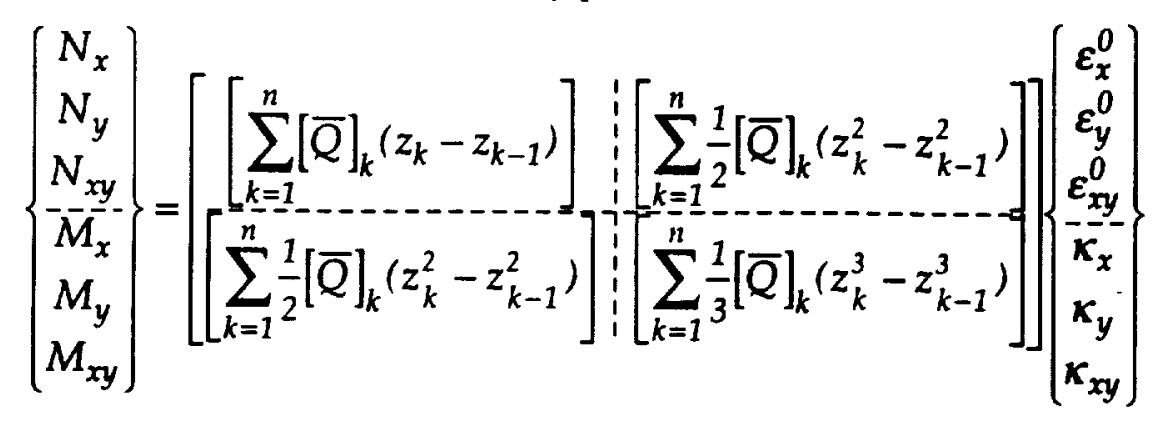

For the remainder of this section, we will be concerned primarily with the A-matrix, the upper left quadrant in (41),

$$
[A]=\sum_{k=1}^{n}[\bar{Q}]_{k}\left(z_{k}-z_{k-1}\right)
$$

since in the case of symmetric laminates and in-plane loading, only this portion of the square matrix in equation (41) is both non-zero and relevant. In this case, the A-matrix leads directly to the calculation of the effective moduli of the laminate. Because we are examining the effects of aging without recourse to a general nonlinear viscoelastic constitutive law, the restriction of constant stress applied to the laminate still applies. Denoting with an overbar the average through the thickness of the stress applied to the laminate, equation (39) reduces to 


$$
\bar{\sigma}_{x}=\frac{N_{x}}{h} ; \bar{\sigma}_{y}=\frac{N_{y}}{h} ; \bar{\sigma}_{x y}=\frac{N_{x y}}{h}
$$

and equation (41) can then be expressed as

$$
\left\{\varepsilon_{x}^{0}(t)\right\}=h[A(t)]^{-1}\left\{\bar{\sigma}_{x}\right\} \equiv[\bar{P}(t)]\left\{\bar{\sigma}_{x}\right\},
$$

where the time dependence of the laminate is now explicitly indicated. Here we take the applied loads to be constant, calculating the time-dependent strain response arising from the time dependence of each lamina as characterized by the S-bar (and thus Q-bar) modulus matrices. As now defined, the P-bar matrix represents the effective modulus matrix of the laminate in the loading coordinate system. For example, using the common notation to let $S_{x}$ denote the effective compliance in the $x$-direction of the laminate

$$
S_{x}(t)=\bar{P}_{11}(t) \text {. }
$$

In this manner, the effects of physical aging on the time dependent response of a laminate to constant stress states can be determined, using the basic concepts of physical aging to express first the transverse and shear compliance of a lamina in the fiber directions, then transforming those expressions to obtain the compliance of each lamina in the $\mathrm{x}$-direction, then building up the overall laminate response through lamination theory on the compliance of each lamina. As an illustration of the results one can obtain with this method, consider a quasi-isotropic laminate, $[0 / \pm 45 / 90]_{S}$. In Figure 16 the change in compliance of this laminate (using the IM7-8320 composite data) is given over a long time range, plotted on a semi-log scale. Note that even though the quasi-isotropic laminate is a fiber dominated lay-up, the compliance changes by $8 \%$ $12 \%$ over a 10 year period. Such a magnitude of modulus change is certainly significant for structural applications and must be considered in the design stages. It should be pointed out here that the effects of physical aging in terms of stiffness of the composite under long-term loading are beneficial: that is, if increase of relaxation times of the matrix was not occurring over time, the long time compliance for the composite would collapse to the momentary curve value, also indicated for $S_{x}$ on Figure 16. In this case, the compliance increases (and the stiffness decreases) at a very rapid rate under constant loading, undesirable for load bearing structures. In view of the 
section on Aging to Equilibrium, where the momentary response is obtained as a long-term response, it is thus also important for composite laminate systems to assure that the equilibrium aging time will never be reached at the times and temperatures of use. The possible adverse effects of physical aging include increased brittleness or decreased fracture toughness with aging (Chen-Chi, Lee et al., 1992).

\section{Conclusion}

The work presented here has outlined a complete analysis of the modeling of physical aging of polymeric composite laminates. The theory for handling aging of polymers was given a much needed review and presented in detail. In addition, the theory for aging to equilibrium (applicable to polymers and polymer composites alike) was presented for the first time, and a new formalization of aging-time and time-temperature relationships was presented. The physical aging theory was built up consistently from the pure polymer case, extending to response of a composite lamina and then finally a general laminate. The methodology can be applied to easily and accurately assess the long-term response of composite systems, considering both the effects of different matrix material properties and different composite lay-ups. A critical feature of the modeling is that the long-term response is calculated based upon several clearly defined parameters that are measured in short-time laboratory tests.

It was demonstrated that the aging shift rate parameter is the most critical parameter in determining magnitude of agings' effect on long-term response. It was also illustrated in the calculation of the response of just a single off-axis lamina, that the interplay of the various timedependent parameters and transformations involved prohibit intuitive guessing of, for example, the "least compliant" fiber orientation. This underscores the need for models such as this one to be able to reasonably predict the long-term response of composites without extensive long-term laboratory testing. It was also demonstrated that even for fiber-dominated lay-ups, physical aging is extraordinarily important in calculating the long-term response of laminates accurately. 


\section{Acknowledoments}

The authors would like to recognize the American Society for Engineering Education - Summer Faculty Fellowship Program and NASA-Langley for their sponsorship of this work.

\section{References}

Brinson, L C., (1990). Time-temperature response of multi-pbase viscoelastic solids tbrough numerical analysis, Ph.D. Thesis, California Institute of Technology.

Chen-Chi, M., C. Lee, M. Chang and N. Tai (1992). Effect of Physical Aging on the Toughness of Carbon Fiber-Reinforced Poly(ether ether ketone) and Poly(phenylene sulfide) Composites. I. Polym. Comp. 13(6), $441-447$.

Ferry, J. D. (1980). Viscoelastic Properties of Polymers, 3rd Edition, 3rd Edition, John Wiley and Sons, Inc., New York.

Findley, W. N., J. S. Lai and K. Onaran (1989). Creep and Relaxation of Nonlinear Viscoelastic Materials, Dover Publications, Inc., New York.

Gates, T. S. and M. Feldman (1993). Time Dependent Behavior of a Graphite/Thermoplastic Composite and the Effects of Stress and Physical Aging. NASA TM-109047, November, 1993.

Halpin, J. C. (1992). Primer on Composite Materials Analysis, 2nd Edition, Technomic Publishing Co., Lancaster.

Hastie, R. L. J. and D. H. Morris (1993). The Effect of Physical Aging on the Creep Response of a Thermoplastic Composite, High Temperature and Environmental Effects on Polymeric Composites, ASTM STP 1174, C.E. Harris and T.S. Gates, Eds., American Society for Testing and Materials, Philadelphia, pp. 163-185.

Jones, R. M. (1975). Mecbanics of Composite Materials, Scripta Book Company, Washington D.C.

Knauss, W. G. and I. J. Emri (1981). Non-Linear Viscoelasticity Based on Free Volume Consideration. Comp. Struct. 13, 123-128.

Kohlrausch, R. (1847). Nachtrag über die Elastische Nachwirkung beim Cocon- und Glasfaden, und die Hygroskopische Eigenschaft des Ersteren. Pogg. Ann. Pbys. 72:11, 393-425.

Lee, A. and G. B. McKenna (1990). The Physical Ageing Response of an Epoxy Glass Subjected to Large Stresses. Polymer 31, 423-430.

Morland, L W. and E. H. Lee (1960). Stress Analysis for Linear Viscoelastic Materials with Temperature Variation. Trans. Soc Rbeol IV, 233-263.

Rusch, K C. (1968). Time-temperature superposition and relaxational behavior in polymeric glasses. $J$. Macromol Sci.Pbys. B2:2, 179-204.

Santore, M. M., R. S. Duran and G. B. McKenna (1991). Volume Recovery in Epoxy Glasses Subjected to Torsional Deformations: the question of rejuvenation. Polymer 32(13), 2377-2381.

Struik, L. C. E. (1978). Pbysical Aging in Amorpbous Polymers and Otber Materials, Elsevier Scientific Publishing Co., New York.

Sullivan, J. L. (1990). Creep and Physical Aging of Composites. Comp. Sa. and Tecbnol 39, 207-232.

Sullivan, J. L., E. J. Blais and D. Houston (1993). Physical Aging in the Creep Behavior of Thermosetting and Themoplastic Composites. Comp. Sai and Tecbnol 47, 389-403. 


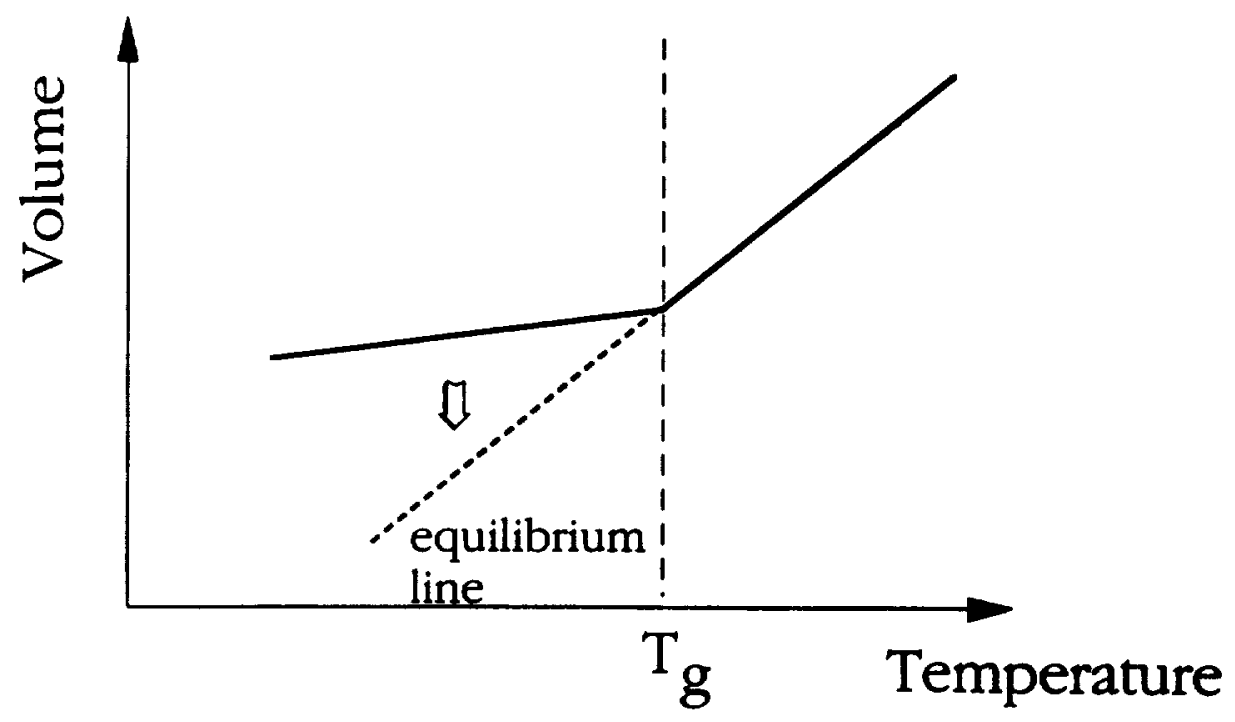

Figure 1: Evolution of volume towards equilibrium with time below the glass transition temperature

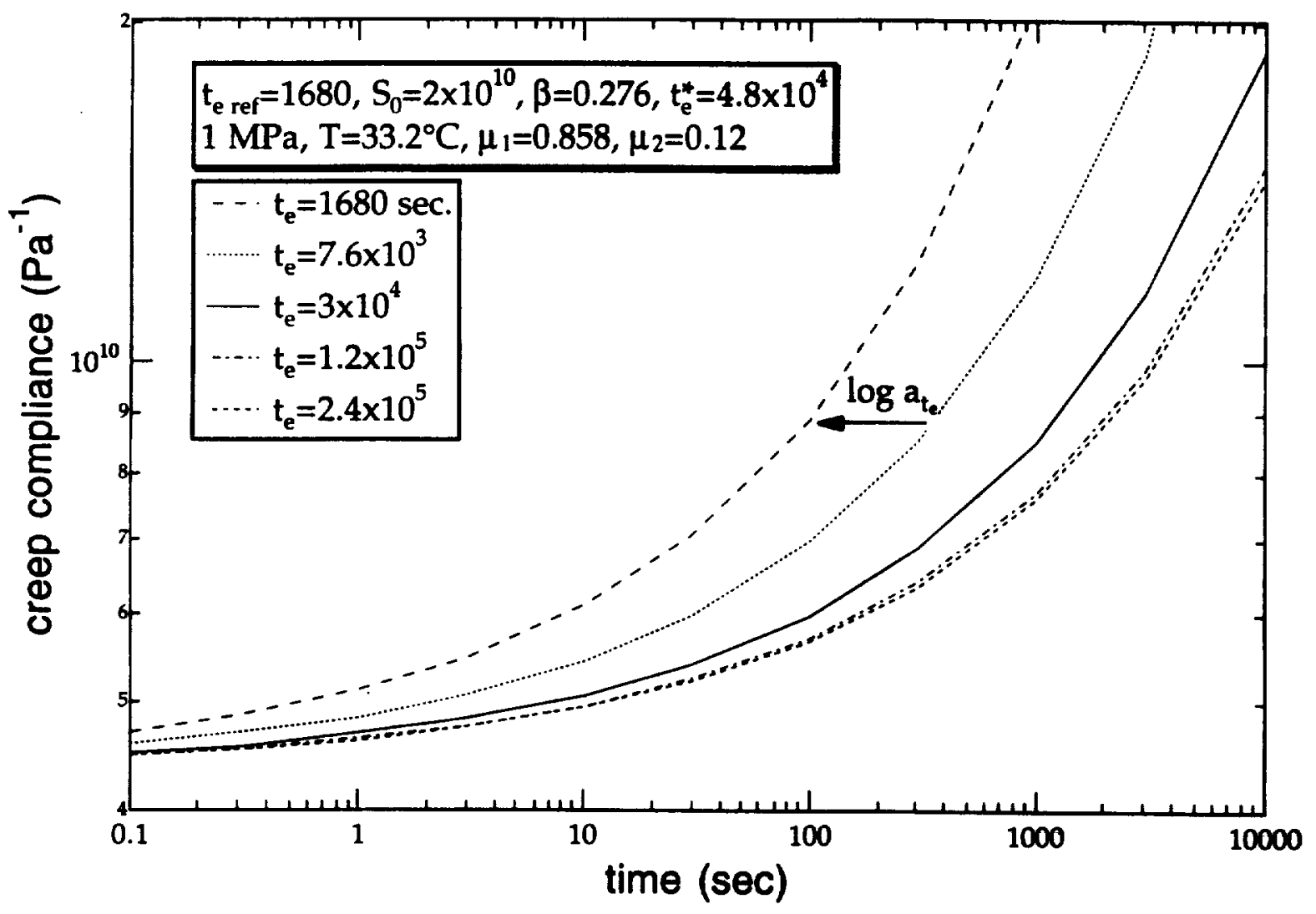

Figure 2a: Momentary creep curves for epoxy with progressing aging time. Experimental data to approximately $10^{10} \mathrm{~Pa}^{-1}$ for each aging time (Lee and McKenna, 1990). Aging to equilibrium phenomenon also illustrated for longer aging times. 


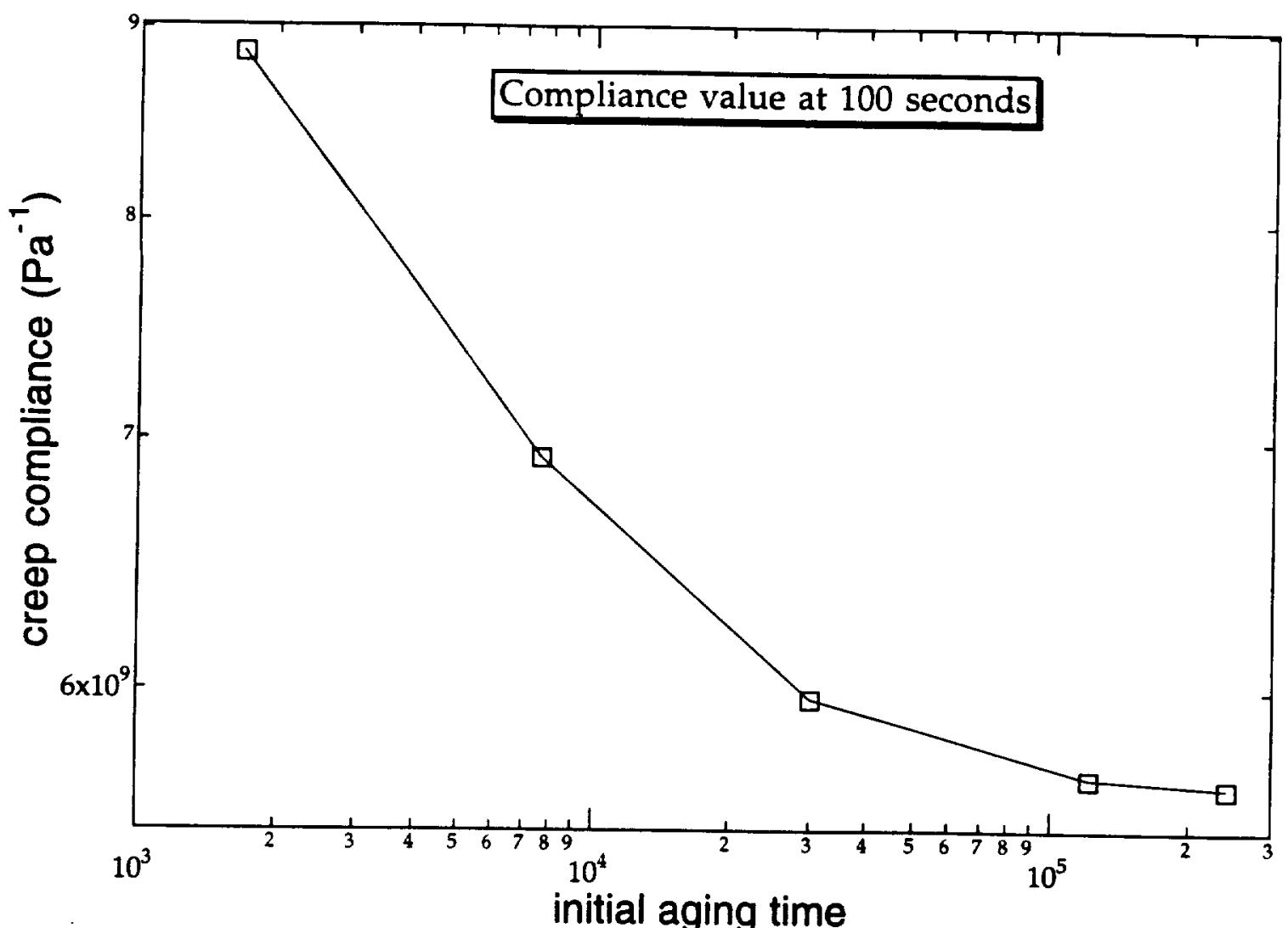

Figure 2b: Isochronal values of compliance from momentary creep curves in Figure 2a versus increasing initial aging times.

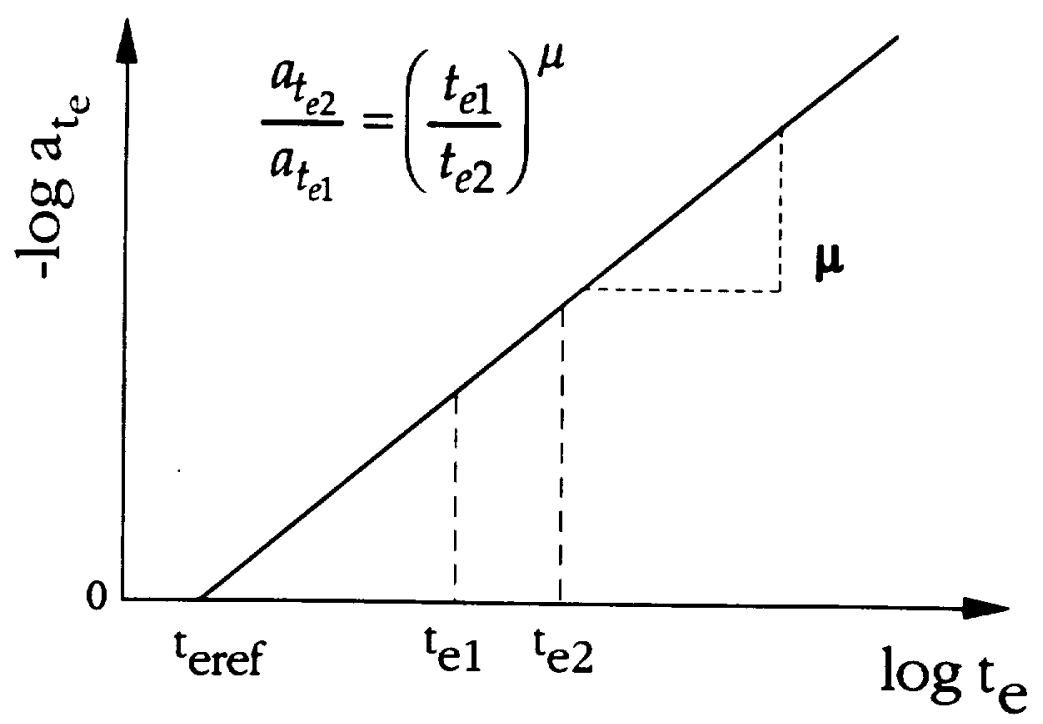

Figure 3: Definition of Shift Rate 


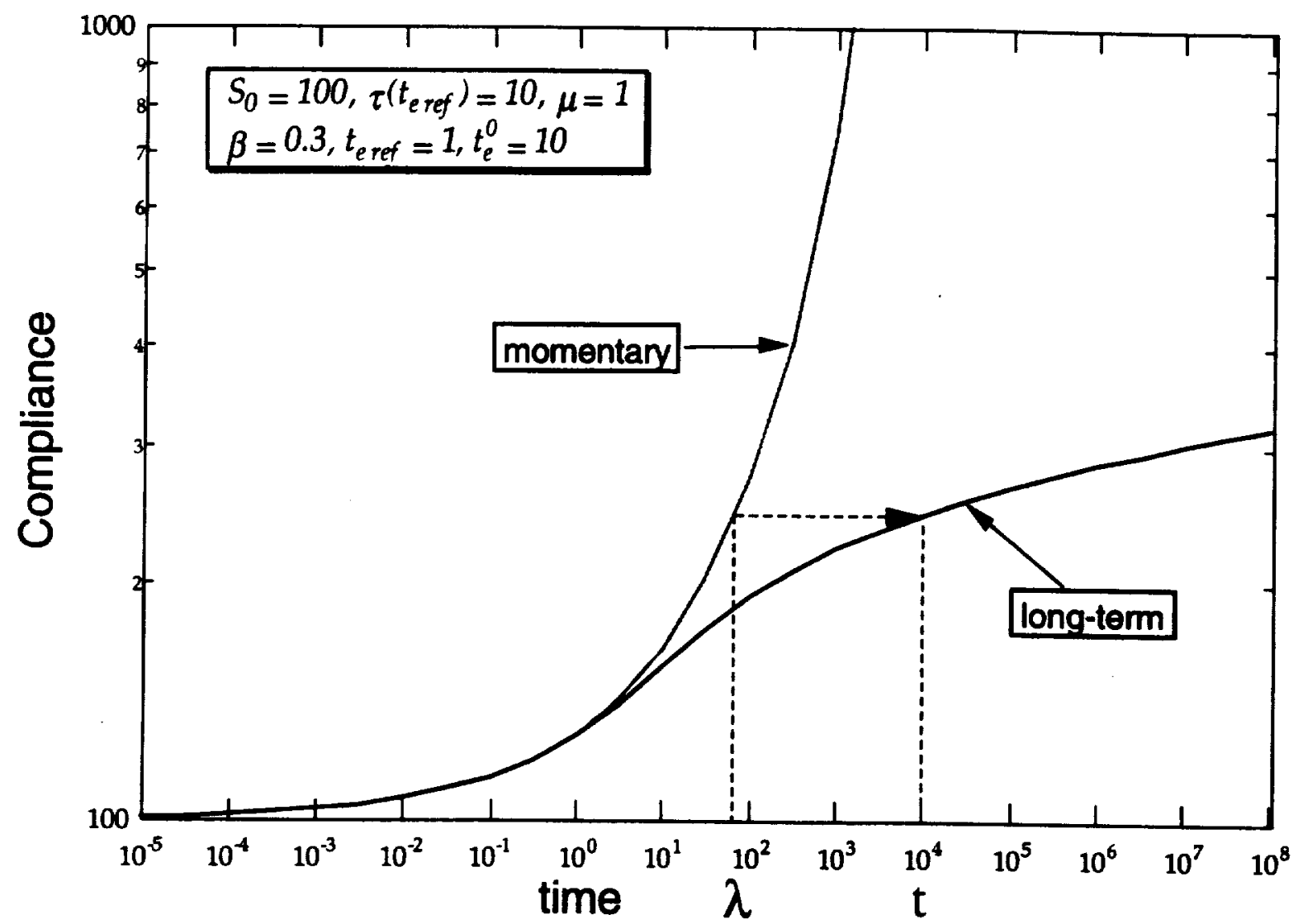

Figure 4: Comparison of long-term and momentary response for polymer with idealized properties 


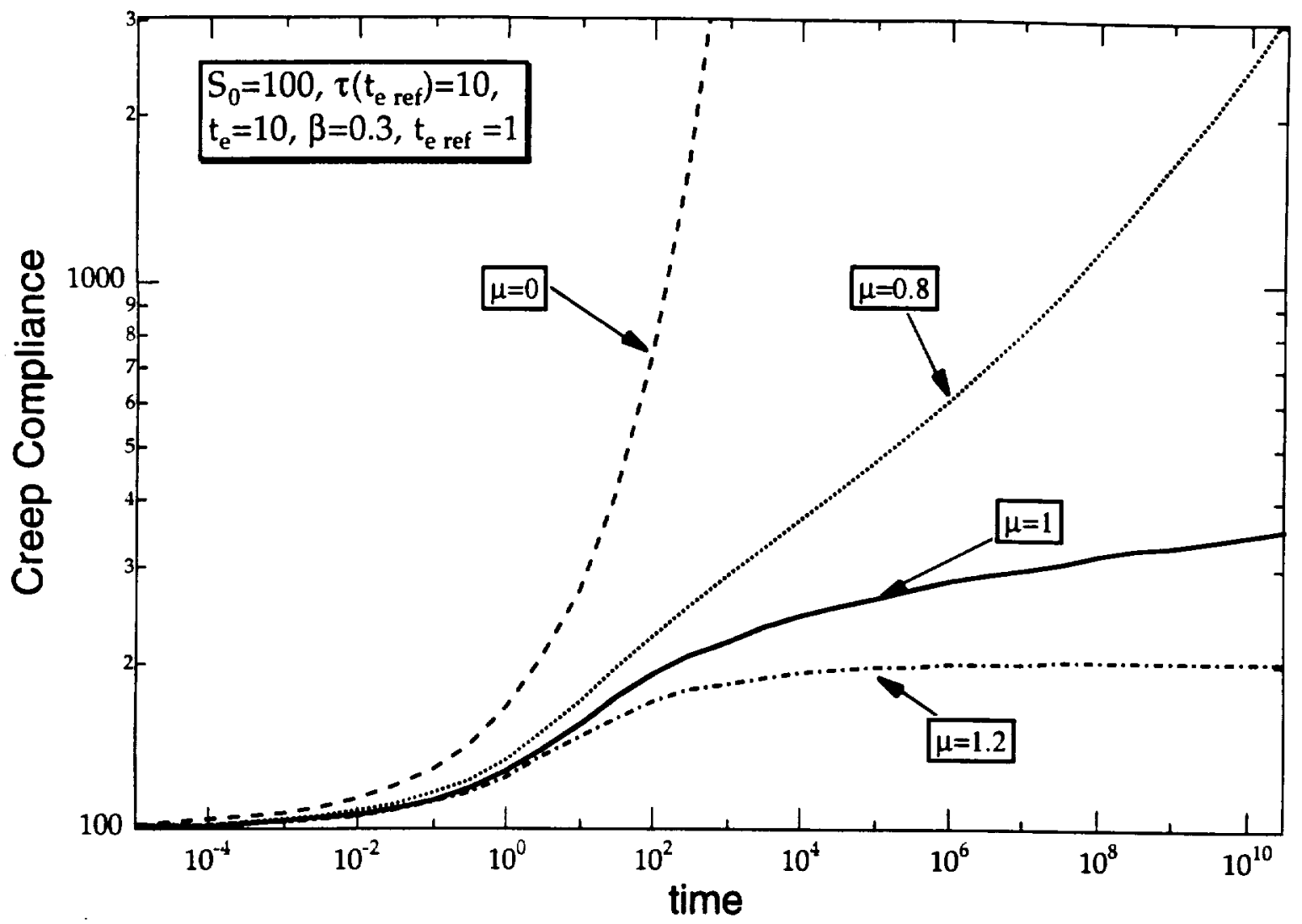

Figure 5: Effect of different shift rates on the long-term material response on polymer with idealized properties

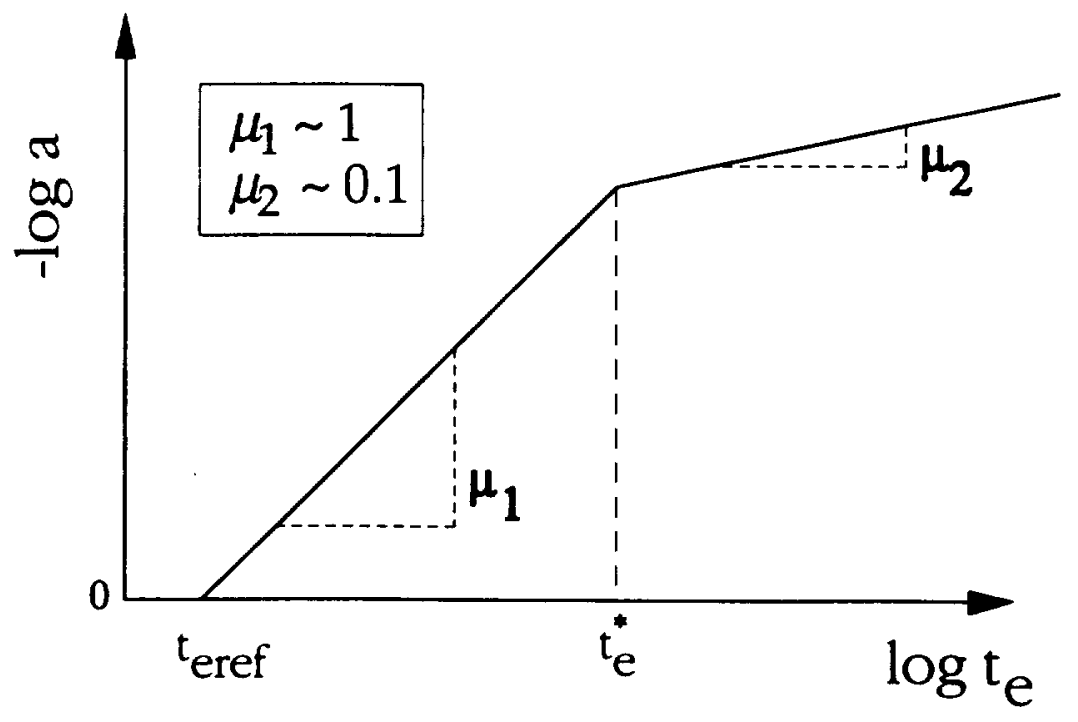

Figure 6: Bilinear shift rate with equilibrium aging time, $t_{e}^{*}$ 


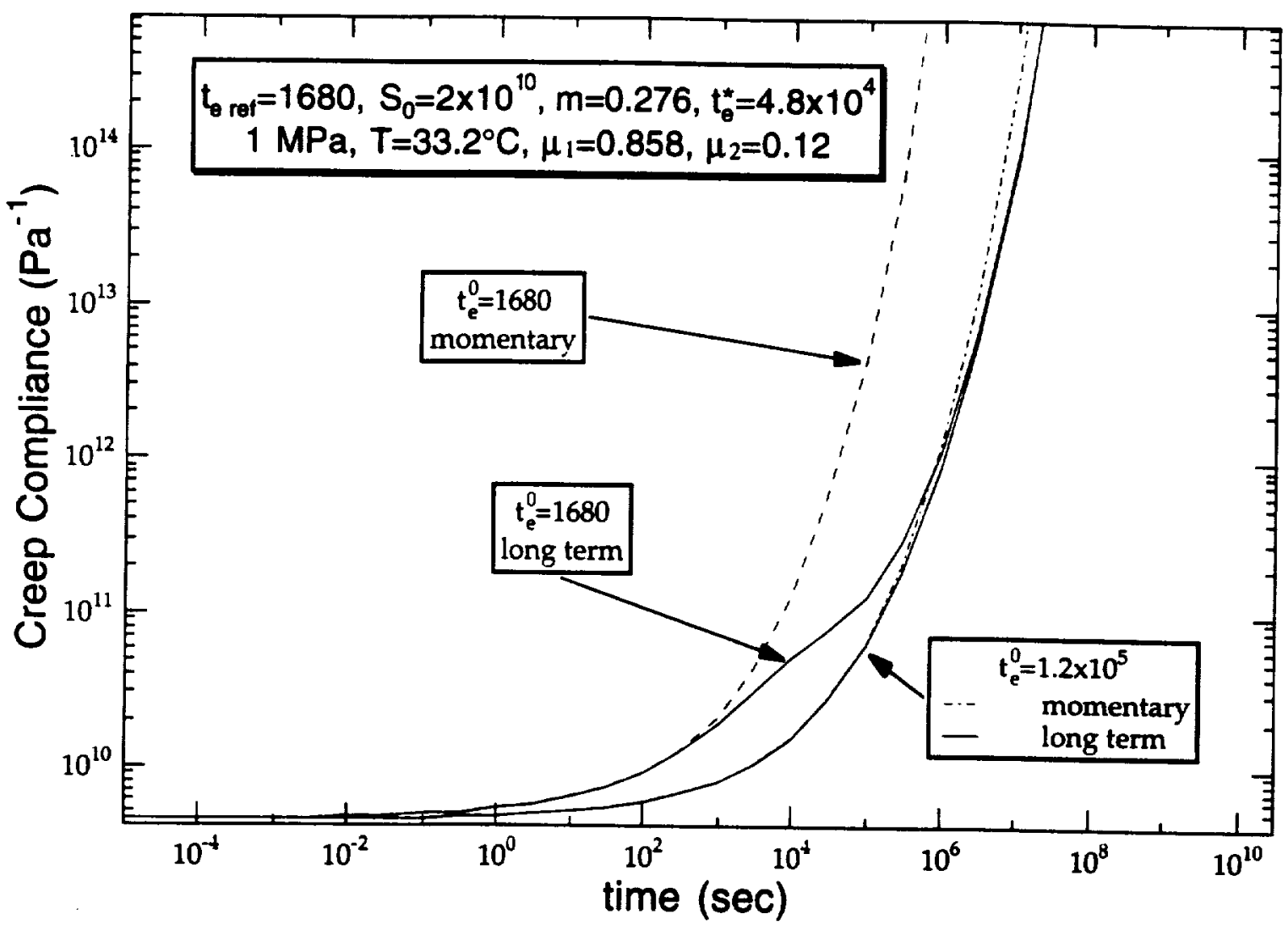

Figure 7: Momentary and long term response when aged to equilibrium; data after Lee and McKenna (1990). 

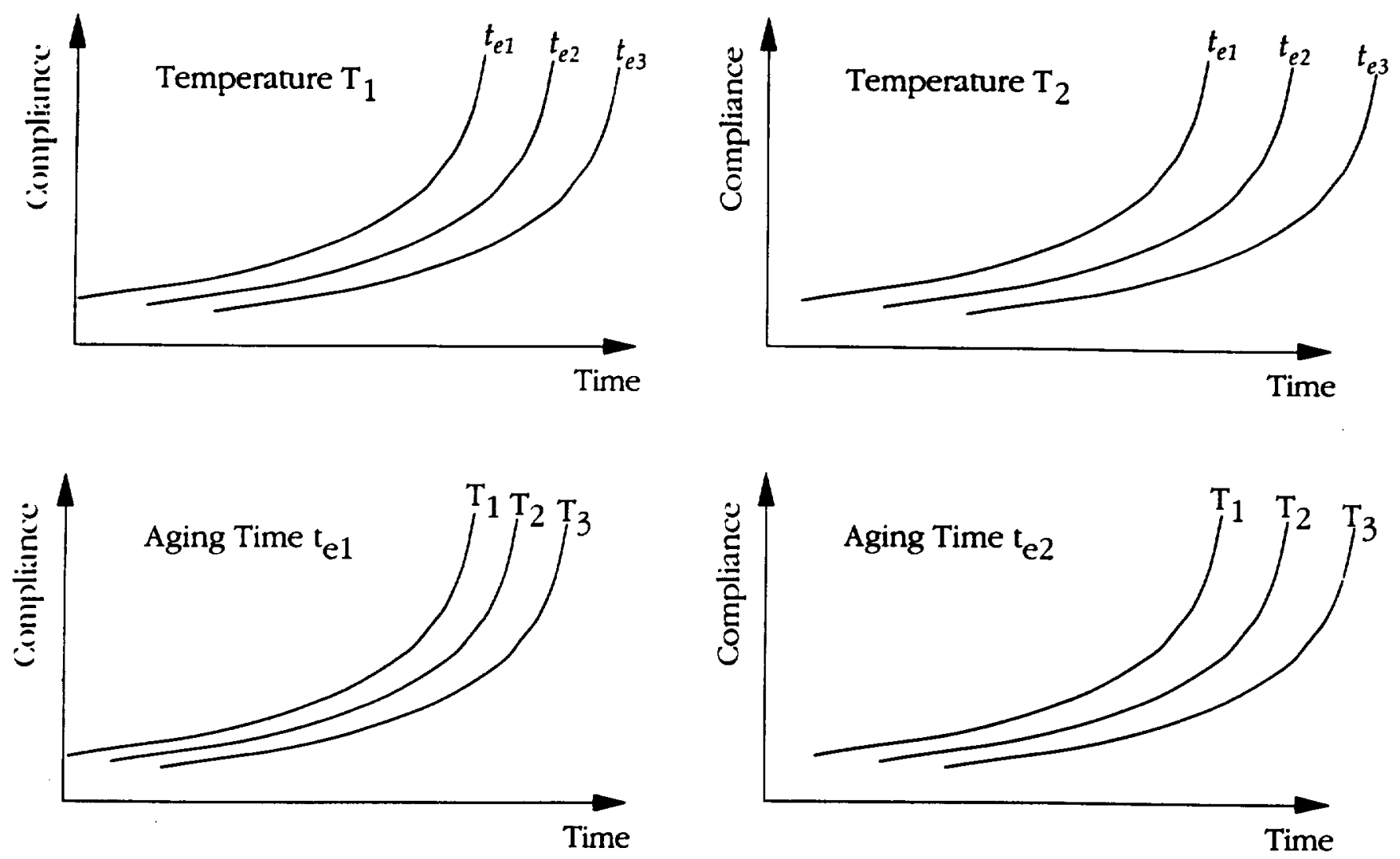

Figure 8: Schematics of compliance curves at two temperatures and two aging times to visualize aging-time and temperature relationship.

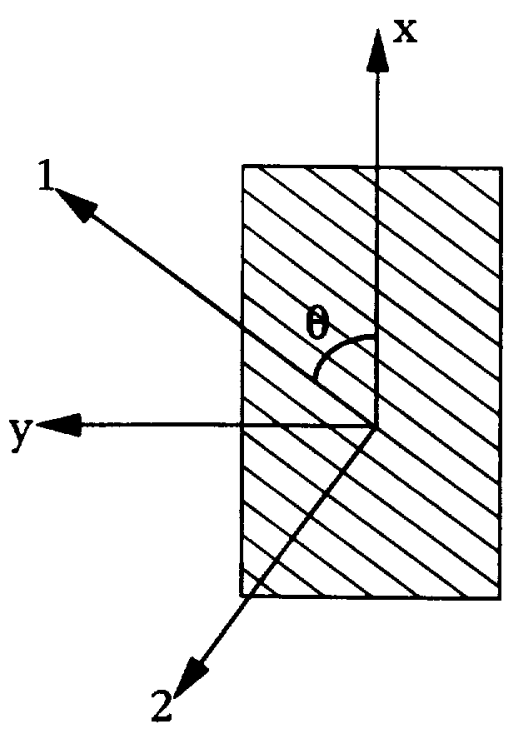

Figure 9: Typical coordinate directions for composite lamina. 


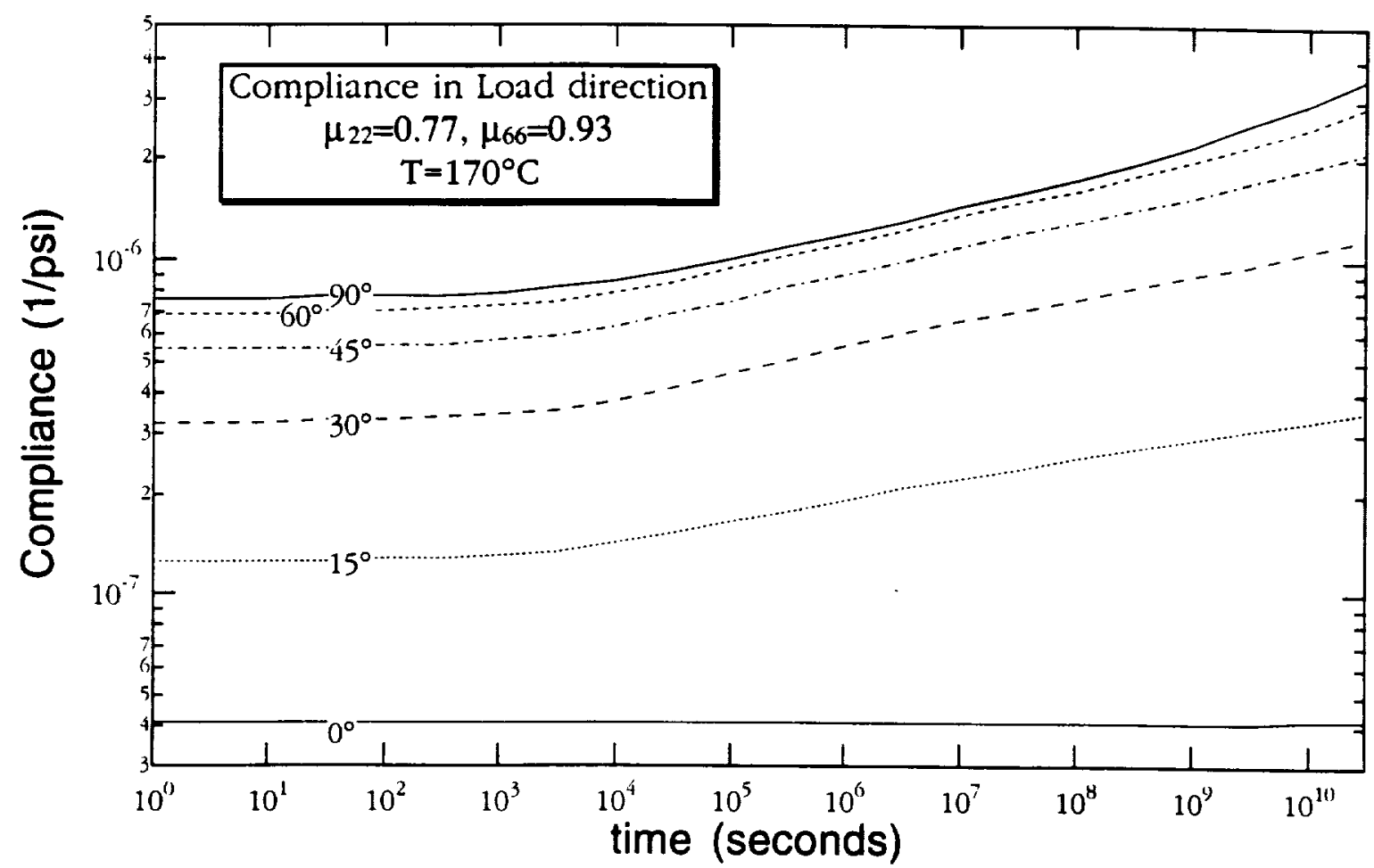

Figure 10: Comparison of the effect of aging on the long term compliance for varying fiber angles. Data on IM7/8320 (Hastie and Morris, 1993).

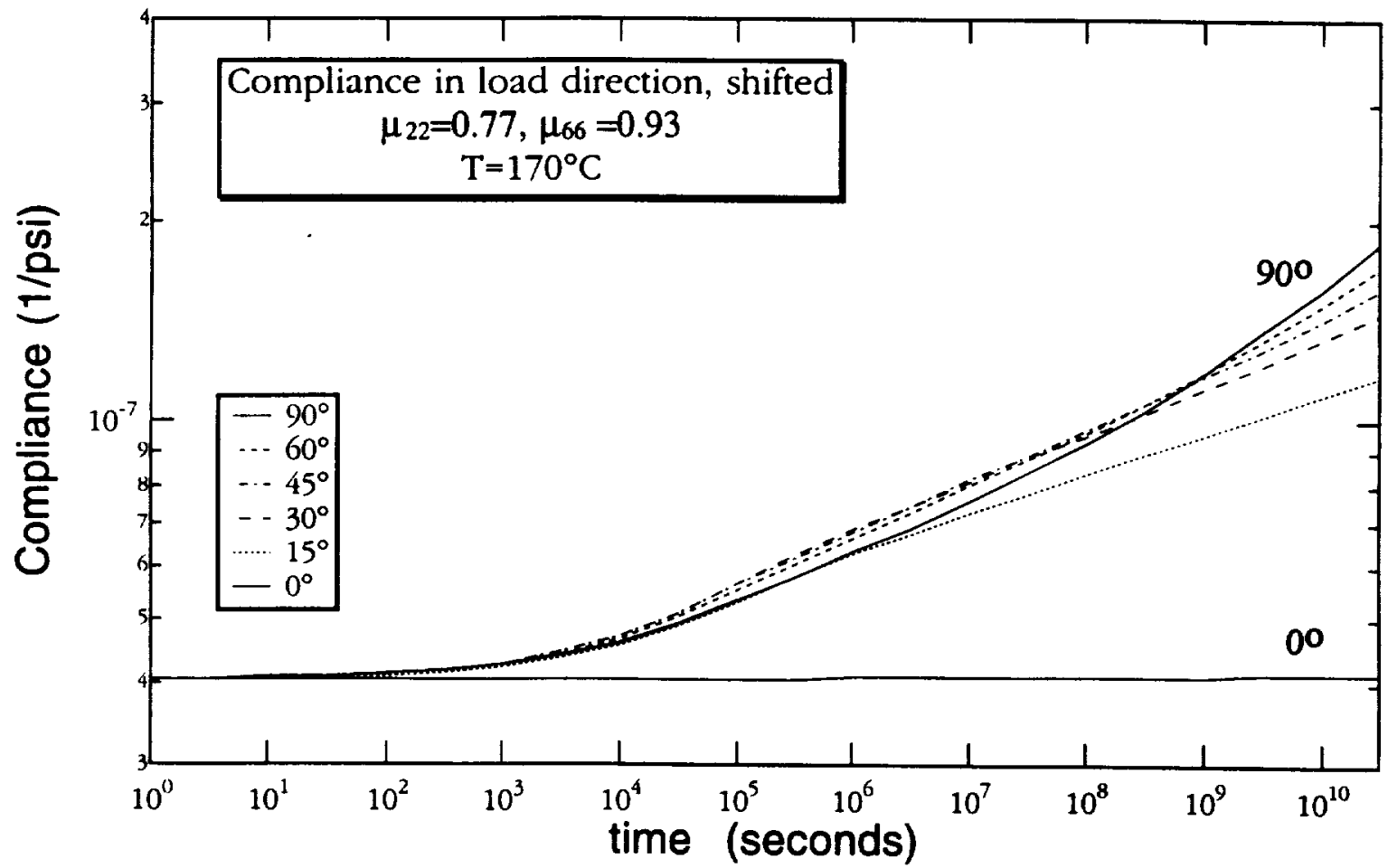

Figure 11: Long-term compliance for various fiber angles all shifted vertically to $0^{\circ}$ curve. Illustrates differing effects of aging. 


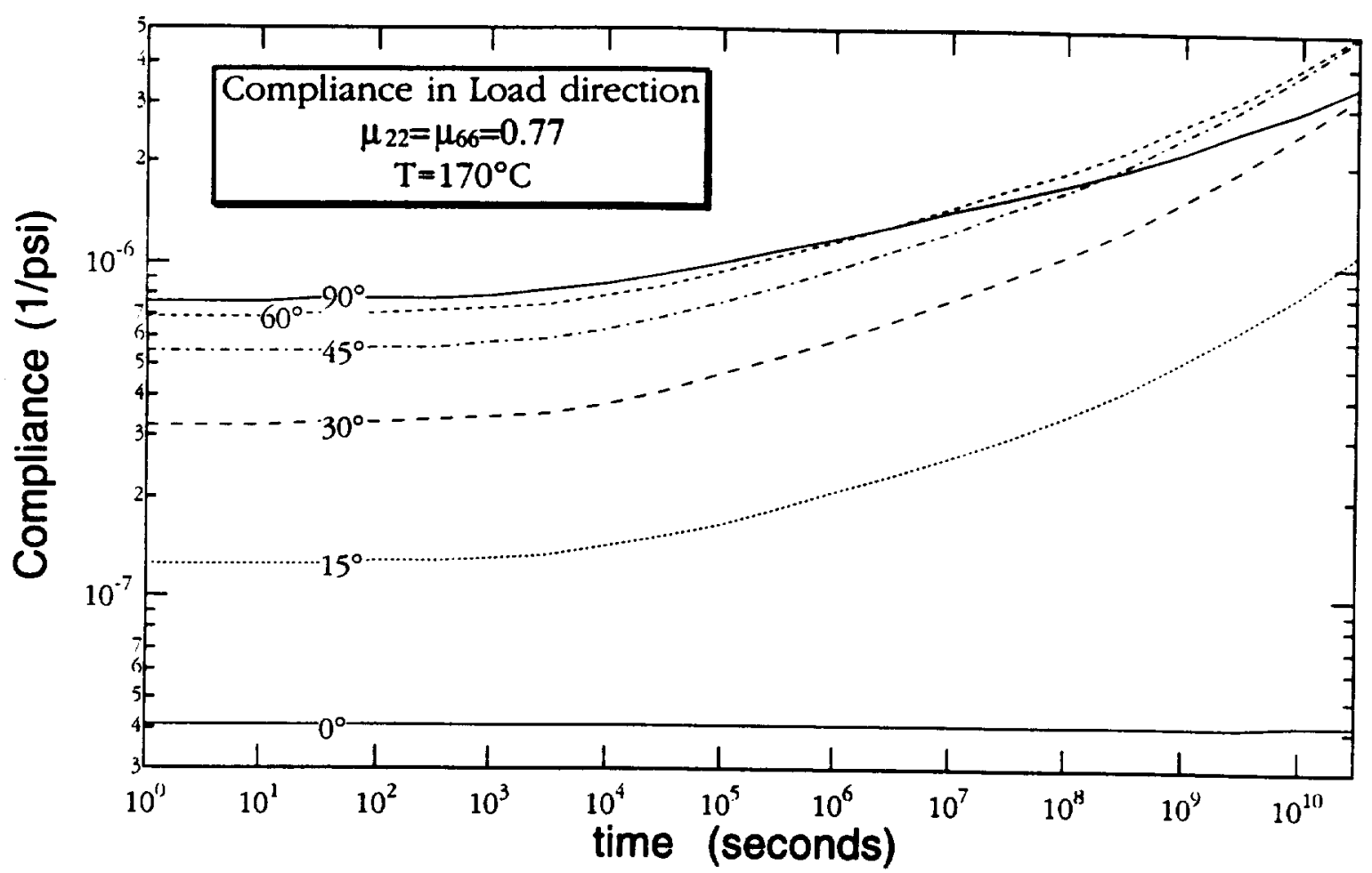

Figure 12: Comparison of the effect of aging on the long term compliance for varying fiber angles. Data on IM7/8320 (Hastie and Morris, 1993), shift rate modified.

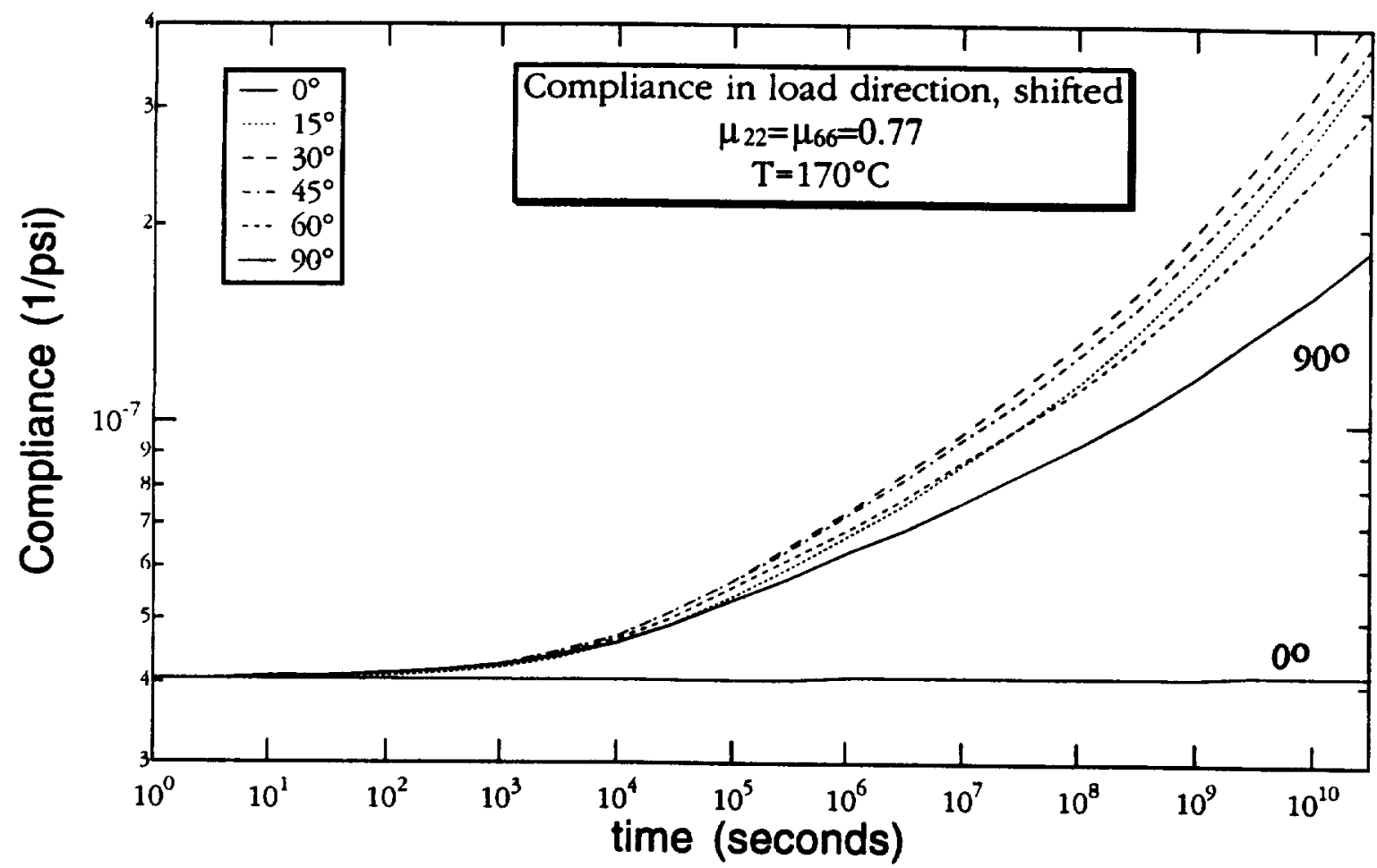

Figure 13: Long-term compliance for various fiber angles all shifted vertically to $0^{\circ}$ curve. Data on IM7/8320 (Hastie and Morris, 1993), shift rate modified. 


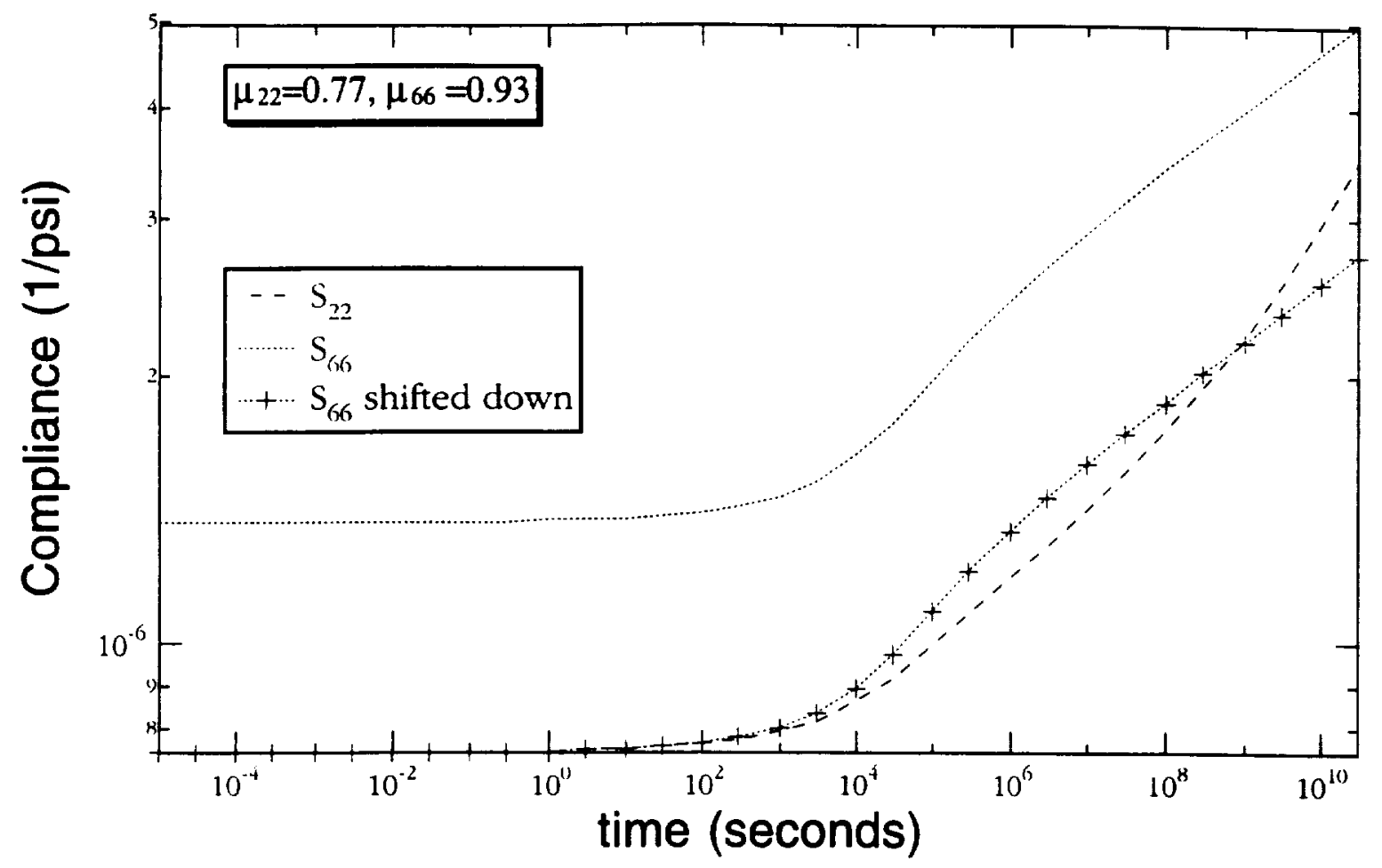

Figure 14: Long-term transverse and shear compliance of IM7/8320 lamina .

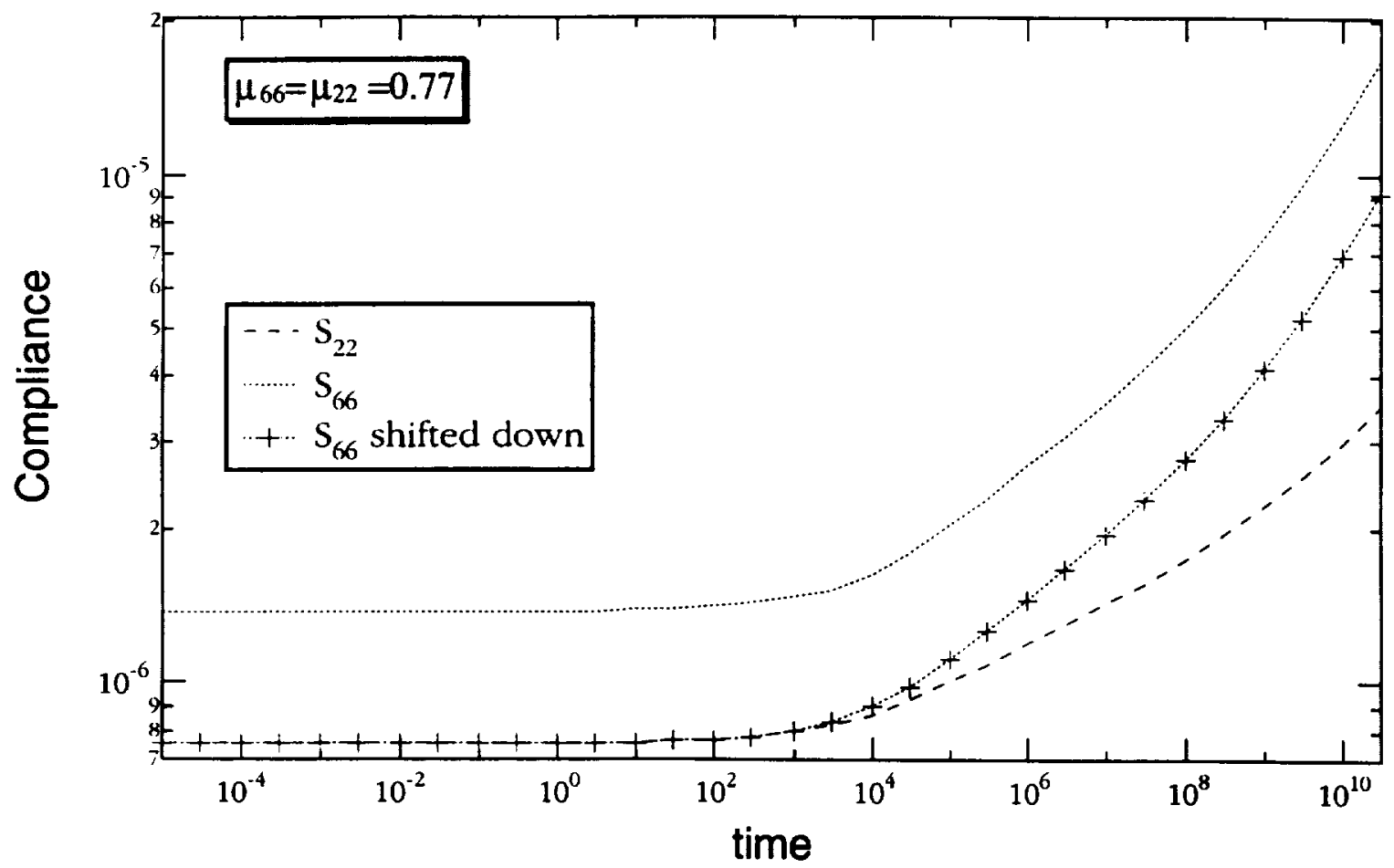

Figure 15: Long-term transverse and shear compliance of IM7/8320 lamina (shift rate modified). 


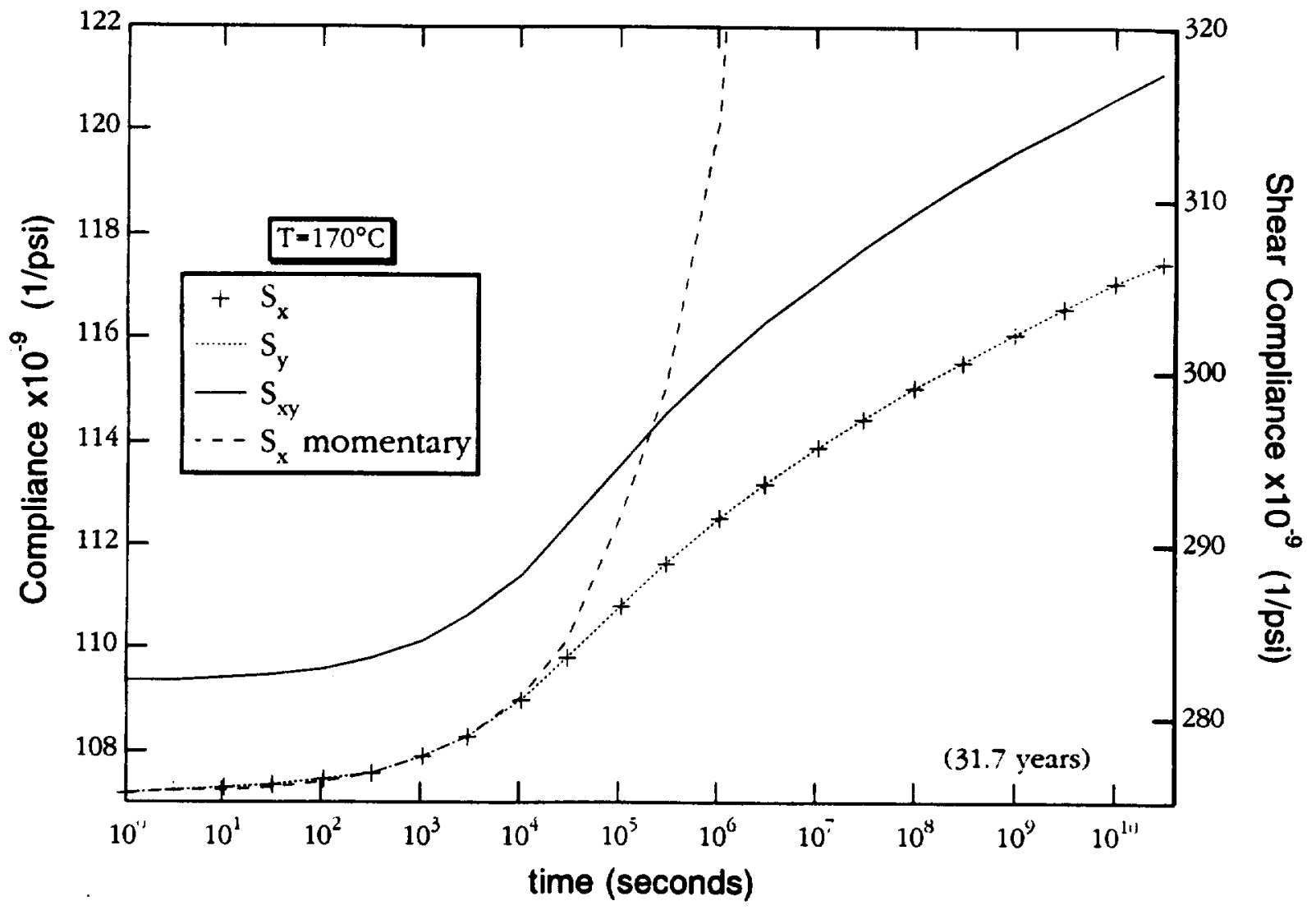

Figure 16: Change in compliance of quasi-isotropic laminate of IM7/8320. 


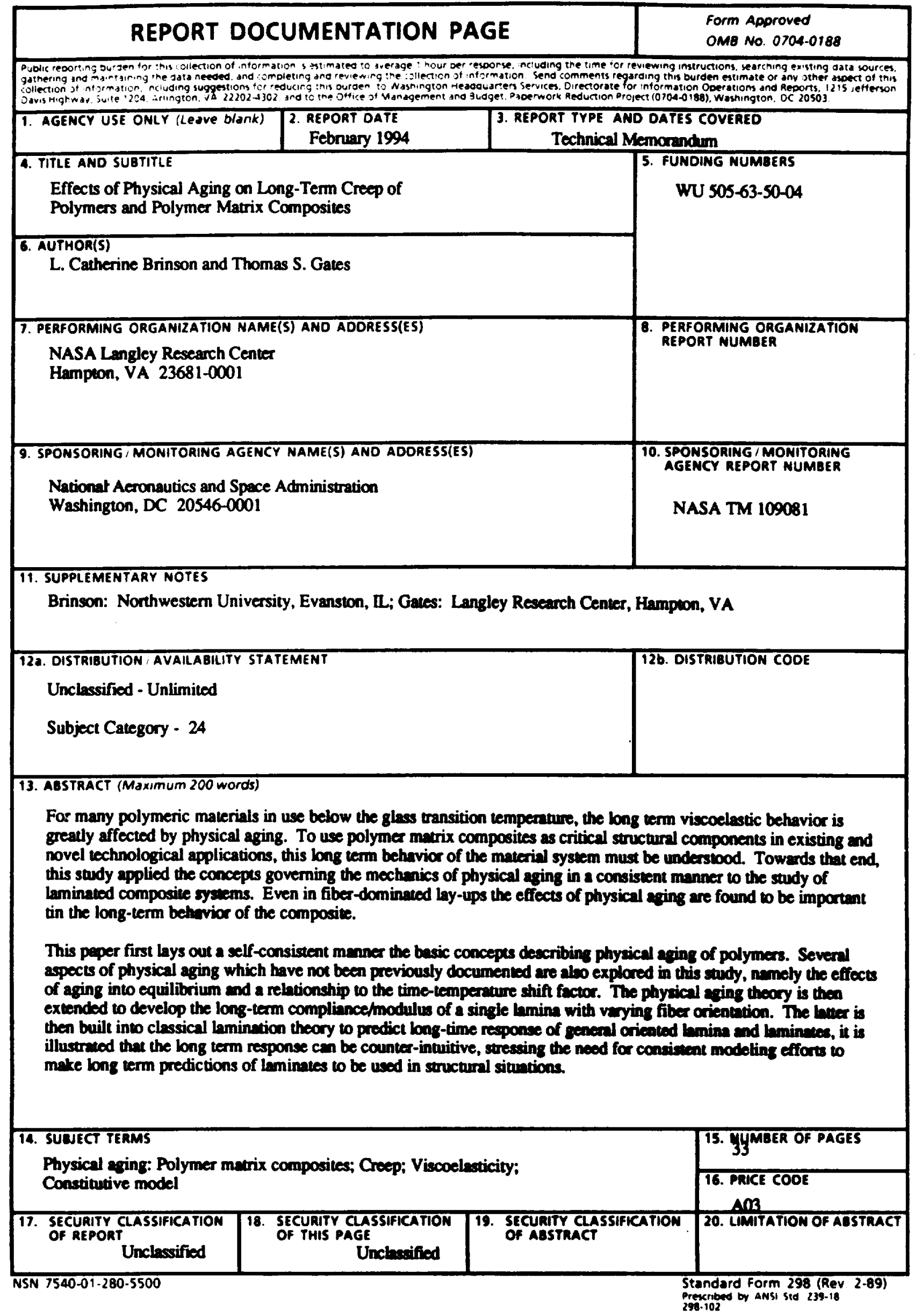

